\title{
Broad-Band HBT BPSK and IQ Modulator MMICs and Millimeter-Wave Vector Signal Characterization
}

\author{
Hong-Yeh Chang, Student Member, IEEE, Tian-Wei Huang, Senior Member, IEEE, Huei Wang, Senior Member, IEEE, \\ Yu-Chi Wang, Pane-Chane Chao, Senior Member, IEEE, and Chung-Hsu Chen
}

\begin{abstract}
Reflection-type binary phase-shift keying and in-phase and quadrature modulator monolithic microwave integrated circuits (MMICs) are reported in this paper. These MMICs are fabricated by $1-\mu \mathrm{m}$ HBT process and evaluated successfully under vector signal characterization. A cold-mode HBT device model with varying bias conditions is proposed, which is suitable for millimeter-wave circuit design and simulation. The analysis and design equations of imbalance effects for the reflection-type modulators are also presented. These MMICs demonstrate measured error vector magnitude of less than $12 \%$, a carrier rejection of better than $15 \mathrm{~dB}$, and an adjacent channel power ratio of better than $-21 \mathrm{dBc}$ from 50 to $110 \mathrm{GHz}$.
\end{abstract}

Index Terms-Binary phase-shift keying (BPSK), cold-mode HBT model, HBT, in-phase and quadrature (IQ), millimeter wave, modulator, monolithic microwave integrated circuit (MMIC).

\section{INTRODUCTION}

$\mathbf{I}$ N-PHASE AND quadrature (IQ) and binary phase-shift keying (BPSK) modulators are the key components for digital communications because they can perform multiformat modulations. A good IQ modulator requires a high linearity especially for high-level modulations. Therefore, most IQ modulators are composed of the doubly balanced mixers [1]-[4] due to superior spur performance, linearity, and port-to-port isolation, but they usually need a high local oscillator (LO) drive power. Alternately, using Si (complementary metal-oxide-semiconductor (CMOS), bipolar junction transistor (BJT), or SiGe HBT) and GaAs [HBT or high-electron mobility transistor (HEMT)] technologies, the IQ modulators based on the Gilbert cell or resistive ring architecture have been reported in recent years [5]-[9]. Although they demonstrated good circuit performance, most of them operated below $10 \mathrm{GHz}$.

Reflection-type BPSK and IQ modulators are based on a reflection-type phase shifter [12], which utilizes a Lange coupler as a $90^{\circ}$ hybrid and two shunt cold-mode devices as switching devices. The cold-mode devices can be HEMT or HBT devices. Some BPSK modulators are implemented in the HEMT process [10]-[13], however, they require negative biasing voltage to perform the modulation function. On the contrary, the HBT-based

Manuscript received June 23, 2003; revised September 10, 2003. This work was supported in part by the National Science Council under Grant NSC 89-2213-E-002-178 and Grant NSC 90-2219-E-002-007 and by the Research Excellence Program funded by the Department of Education of the Republic of China under Grant ME-89-E-FA06-2-4-6.

H.-Y. Chang, T.-W. Huang, and H. Wang are with the Department of Electrical Engineering and Graduate Institute of Communication Engineering, National Taiwan University, Taipei 106, Taiwan, R.O.C.

Y.-C. Wang, P.-C. Chao, and C.-H. Chen are with the WIN Semiconductors Corporation, Taoyuan 333, Taiwan, R.O.C.

Digital Object Identifier 10.1109/TMTT.2004.823573 modulators only require positive bias to operate, but they have been reported in literatures [13], [14] with operating frequencies below $50 \mathrm{GHz}$. Recently, we reported an HBT-based BPSK modulator operating above $50 \mathrm{GHz}$ with a low LO power drive [15]. Using this BPSK modulator in our IQ modulator design, this IQ MMIC chip demonstrated a superior broad-band performance [16].

Recently, we have reported a vector signal characterization system for a millimeter-wave (MMW) IQ modulator [16]. To the best of our knowledge, this is the first attempt of vector signal characterization up to $110 \mathrm{GHz}$ for IQ modulator MMICs. Some MMW vector signal measurements can be found in [3] and [4], which demonstrated a vector signal characterization system with error vector magnitude (EVM) and adjacent channel power ratio (ACPR) measurement results for a power amplifier module at 38 GHz. EVM measurements and direct IQ modulator design issues were reported in the microwave range [8], [9]. For a real digital modulated signal coming from an IQ modulator, the amplitude and phase states are time-variant. Therefore, the amplitude and phase states of digital modulated signals are difficult to measure via a network analyzer or a scalar spectrum analyzer. However, we can use a vector signal analyzer to evaluate the performance of the IQ modulator, such as EVM, phase error, amplitude error, quadrature error, IQ imbalance, dc offset, and the impairment of an imperfect LO source. Additionally, the sideband suppression and carrier suppression measurements are usually used to determine the phase/amplitude error and dc offset for the IQ modulators [6]-[9]. By using a power series for cold-mode HBT, the equations of carrier suppression and sideband suppressions are investigated for reflection-type IQ modulators. Also, the sideband/carrier suppression measurements are performed and compared with the vector signal characterization results.

Cold-mode HBT device modeling can be found in [17], which presented the method of direct extraction of a hybrid-T equivalent circuit for HBT as well as the extraction of extrinsic elements at the ON and OFF states via open collector condition. The proposed model in this paper can predict the MMW characteristics for HBT devices operated under cold-mode, especially with varying base bias. By applying this model to circuit simulation, good agreement with the measurement results has been achieved. The parameters of the HBT model in this paper can be extracted with some hybrid- $\pi$ model methods [18]-[20].

The imbalance analysis and discussions for diode-based IQ modulators can be found in the literature [2], [9], which focus on phase-error compensation and phase/gain imbalance in I and $\mathrm{Q}$ paths. However, the reflection-type modulators are composed of broad-band couplers and cold-mode devices. The imbalance 


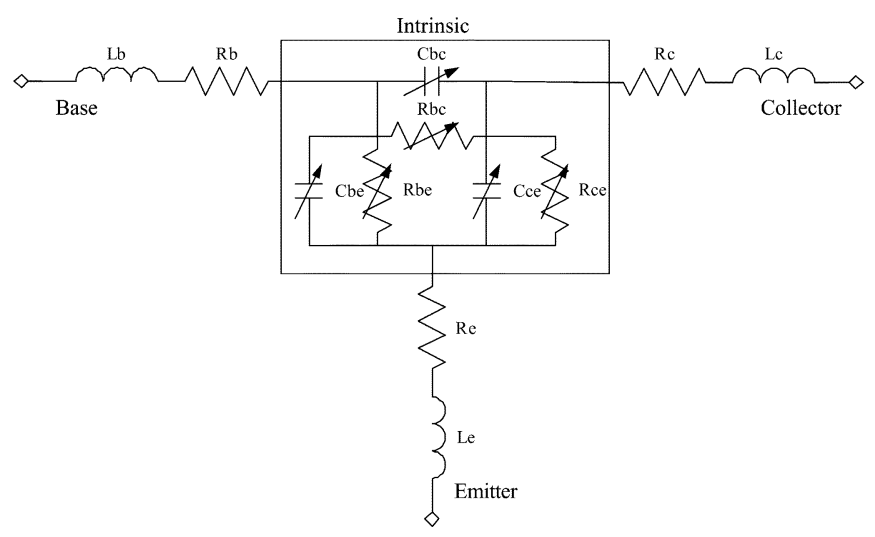

Fig. 1. Equivalent circuit of the cold-mode HBT device.

effects of reflection-type came from the amplitude/phase imbalances of couplers and devices. In this paper, the imbalance analysis for reflection-type modulators and the design rules are also developed.

This paper is organized as follows. Section II shows the MMIC process for the modulator circuit design and a device model for the cold-mode HBT. Section III describes imbalance analysis for the reflection-type modulator. Section IV gives experimental results and characterization. Conclusions are given in Section V.

\section{MMIC PRocess AND Device Modeling}

These broad-band modulators are fabricated using a 6-in 1- $\mu \mathrm{m}$ GaAs HBT MMIC process on a 4-mil substrate provided by the WIN Semiconductors Corporation, Taoyuan, Taiwan, R.O.C. [21]. A single-emitter-finger HBT device with an emitter size of $1.4 \times 10 \mu \mathrm{m}^{2}$ is selected for the modulator designs. The equivalent circuit used for the HBT device is a $\pi$-model, as shown in Fig. 1. This model consists of parasitic elements and intrinsic elements. The parasitic elements are bias-independent, while, the intrinsic elements are bias-dependent within the solid box, as shown in Fig. 1. The extraction of parasitic elements can be determined via open-collector bias condition [17]. At high base current, the resistance of the base-emitter junction and base-collector junction is low. We can use $Z$-parameters to extract the parasitic resistances and inductances directly [19]. The extracted results of the parasitic element for an emitter size of $1.4 \times 10 \mu \mathrm{m}^{2}$ are shown in Table I.

The extraction of the intrinsic elements can be carried out by calculating the $Y$-parameters of the solid box in Fig. 1. The extracted results of intrinsic elements for OFF-state operation are shown in Table II. There are two configurations in our circuit design for ON-state operation: open collector and a collector biased with a $50-\Omega$ resistor, as shown in Fig. 2. The extracted results of intrinsic elements for the ON state are also shown in Table II. It can be observed from Table II that the intrinsic elements are slightly different between these two configurations when the frequency is below $50 \mathrm{GHz}$. Based on the device models, the reflection coefficients of these two configurations will have significant differences for higher frequency operation in the circuit simulations due to the difference of the intrinsic capacitance and the effect of parasitic elements. The magnitude difference of re- flection coefficients between the ON and OFF states became large as the frequency approached $100 \mathrm{GHz}$.

The extracted $C_{\mathrm{be}}, C_{\mathrm{bc}}$, and $C_{\mathrm{ce}}$ at the several bias conditions for $I_{b}$ and $I_{c}=0 \mathrm{~mA}$ (open collector) are plotted in Fig. 3 . Using the curve-fitting method for the extracted results, we derived the expressions for these bias-dependent capacitances as follows:

$$
\begin{aligned}
C_{\mathrm{be}}\left(I_{b}\right)= & 4.2+19.4 \exp \left[\frac{-\left(I_{b}+0.08\right)}{0.39}\right] \\
C_{\mathrm{bc}}\left(I_{b}\right)= & 34+200\left[1-\exp \left(\frac{-I_{b}}{1.97}\right)\right] \\
& +121\left[1-\exp \left(\frac{-I_{b}}{0.066}\right)\right] \\
C_{\mathrm{ce}}\left(I_{b}\right)= & 8+60.6\left[1-\exp \left(\frac{-I_{b}}{1.28}\right)\right] \\
& +10.6\left[1-\exp \left(\frac{-I_{b}}{8.9 \cdot 10^{-6}}\right)\right] .
\end{aligned}
$$

With the same bias conditions, the extracted conductance of $1 / R_{\mathrm{be}}, 1 / R_{\mathrm{bc}}$, and $1 / R_{\mathrm{ce}}$ are plotted in Fig. 4 . The expressions for these bias-dependent resistances were also derived as

$$
\begin{aligned}
\frac{1}{R_{\mathrm{be}}}\left(I_{b}\right)= & 0.24-\frac{0.249}{1+\exp \left(\frac{I_{b}-4.26}{1.34}\right)} \\
\frac{1}{R_{\mathrm{bc}}}\left(I_{b}\right)= & 1.4 \cdot 10^{-4}+0.2\left[1-\exp \left(\frac{-I_{b}}{2}\right)\right] \\
& +1.17 \cdot 10^{13}\left[1-\exp \left(\frac{-I_{b}}{8.58 \cdot 10^{14}}\right)\right] \\
\frac{1}{R_{\mathrm{ce}}}\left(I_{b}\right)= & 3 \cdot 10^{-5}+0.11\left[1-\exp \left(\frac{-I_{b}}{6.36}\right)\right] \\
& +1.28 \cdot 10^{12}\left[1-\exp \left(\frac{-I_{b}}{4.19 \cdot 10^{14}}\right)\right] .
\end{aligned}
$$

Applying the extracted results in a circuit simulation software (Agilent Advanced Design System software), we obtained a user-defined model for an emitter size of a $1.4 \times 10-\mu \mathrm{m}^{2} \mathrm{HBT}$ device. The measured and simulated $S_{22}$ 's are plotted in Fig. 5. It is observed that they agree well for both the ON and OFF states. However, this HBT model can only be used for cold-mode operation and does not follow the scaling rule with the emitter area. The modeling procedure for cold-mode HBT device is summarized as follows:

1) use $Z$-parameters to extract the parasitic elements [19];

2) obtain the $S$-parameters of intrinsic elements by using de-embedding method;

3) use $Y$-parameters to extract the intrinsic elements;

4) determine the nonlinear expressions for the intrinsic elements by using curve-fitting method;

5) apply overall extracted results to a user-defined model in a circuit simulator.

\section{IMBALANCE ANALYSIS AND CiRCUIT DESIGN}

\section{A. BPSK Modulator}

The BPSK modulator employs two bi-phase reflection modulators [12] operating in push-pull mode using differential control signals. A typical bi-phase reflection modulator is shown in 
TABLE I

EXTRACTED RESUlts of PARASITIC ELEMENTS FOR THE EMITTER Size OF $1.4 \times 10 \mu \mathrm{m}^{2}$

\begin{tabular}{c|c|c|c|c|c}
\hline $\mathrm{L}_{\mathrm{b}}$ & $\mathrm{L}_{\mathrm{e}}$ & $\mathrm{L}_{\mathrm{c}}$ & $\mathrm{R}_{\mathrm{b}}$ & $\mathrm{R}_{\mathrm{e}}$ & $\mathrm{R}_{\mathrm{c}}$ \\
\hline $15.9 \mathrm{pH}$ & $8.7 \mathrm{pH}$ & $8 \mathrm{pH}$ & $4 \Omega$ & $5 \Omega$ & $5.5 \Omega$ \\
\hline
\end{tabular}

TABLE II

EXTRACTED RESULTS OF INTRINSIC ELEMENTS FOR ON-AND OFF-STATE OPERATION

\begin{tabular}{c|c|c|c}
\hline Condition & Off-sate & $\begin{array}{c}\text { On-state at open } \\
\text { collector }\end{array}$ & $\begin{array}{c}\text { On-state at collector } \\
\text { biased with a } 50-\Omega\end{array}$ \\
\hline $\mathrm{R}_{\mathrm{be}}$ & $10 \mathrm{~K} \Omega$ & $7 \Omega$ & $13 \Omega$ \\
\hline $\mathrm{C}_{\mathrm{be}}$ & $20 \mathrm{fF}$ & $77 \mathrm{fF}$ & $15 \mathrm{fF}$ \\
\hline $\mathrm{R}_{\mathrm{bc}}$ & $10 \mathrm{~K} \Omega$ & $4 \Omega$ & $5.2 \Omega$ \\
\hline $\mathrm{C}_{\mathrm{bc}}$ & $34 \mathrm{fF}$ & $343 \mathrm{fF}$ & $319 \mathrm{fF}$ \\
\hline $\mathrm{R}_{\mathrm{ce}}$ & $10 \mathrm{~K} \Omega$ & $13 \Omega$ & $15 \Omega$ \\
\hline $\mathrm{C}_{\mathrm{ce}}$ & $8 \mathrm{fF}$ & $82 \mathrm{fF}$ & $13 \mathrm{fF}$ \\
\hline
\end{tabular}

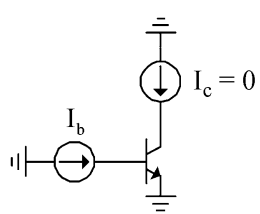

(a)

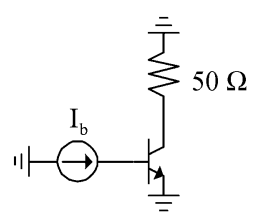

(b)
Fig. 2. Bias configurations. (a) Open collector. (b) Collector biased with a $50-\Omega$ resistor.

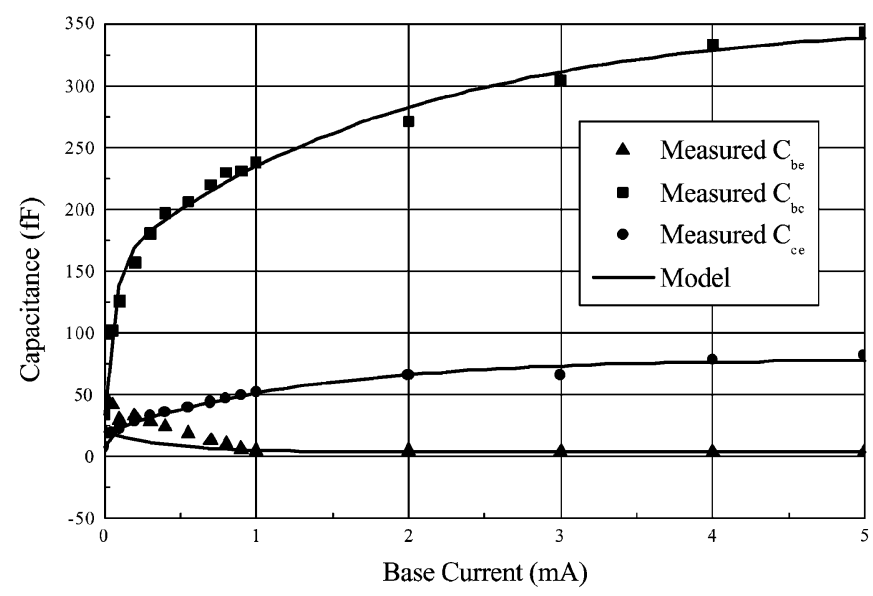

Fig. 3. Measured and model-calculated capacitance of $C_{\mathrm{be}}, C_{\mathrm{bc}}$, and $C_{\mathrm{ce}}$ of a $1.4 \times 10-\mu \mathrm{m}^{2} \mathrm{HBT}$ device for $I_{b}=0,25,50,100$, $200,300,400,550,700,800$, and $900, \mu \mathrm{A}$ and $1,2,3,4$, and $5 \mathrm{~mA}$.

Fig. 6. It consists of a Lange coupler and two cold-mode devices, which are connected to the coupled port and the direct port. The phase difference between coupled port and direct port is $90^{\circ}$ as the phase imbalance is zero. The amplitude imbalance means the amplitude difference between coupled and direct ports. We assume that the coupler has a phase imbalance of $\theta_{e}$ and an amplitude imbalance of $A_{e}\left(A_{e} \geq 1\right.$, while $A_{e}=1$ for perfect balanced case). The transmission coefficients $S_{21}$ of the bi-phase reflection modulator can be expressed as

$$
S_{21}=\frac{\Gamma_{I}}{A_{e}} e^{j\left(\pi / 2+\theta_{e}\right)}
$$

where $\Gamma_{I}$ is the input reflection coefficient of the HBT.

The schematic of the BPSK modulator is shown in Fig. 7(a), where VP and VN are the control voltage ports. The microphotograph of the BPSK MMIC is shown in Fig. 7(b) with a chip

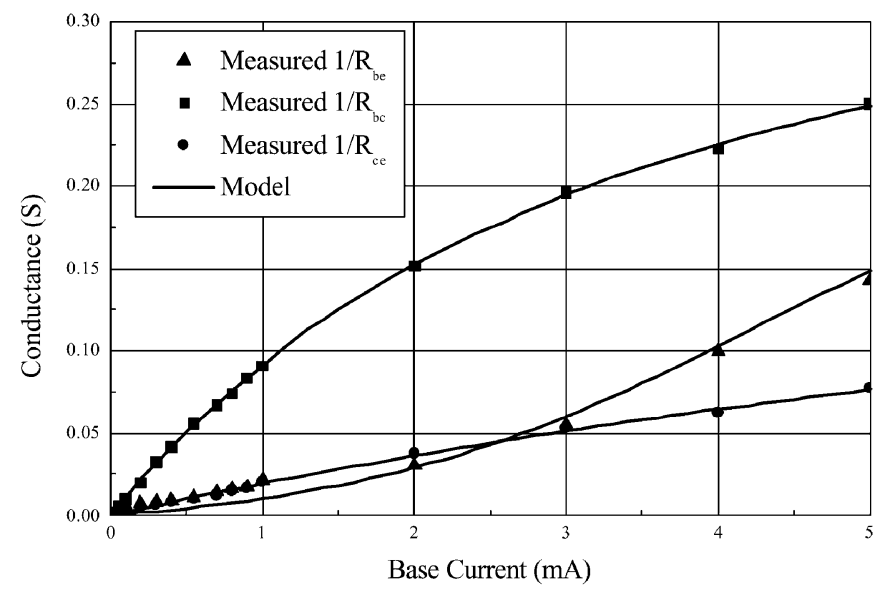

Fig. 4. Measured and model-calculated conductance of $R_{\mathrm{be}}, R_{\mathrm{bc}}$, and $R_{\mathrm{ce}}$ of a $1.4 \times 10-\mu \mathrm{m}^{2}$ HBT device for $I_{b}=\{0,25,50,100$, $200,300,400,550,700,800$, and $900, \mu \mathrm{A}$ and $1,2,3,4$, and $5 \mathrm{~mA}\}$.

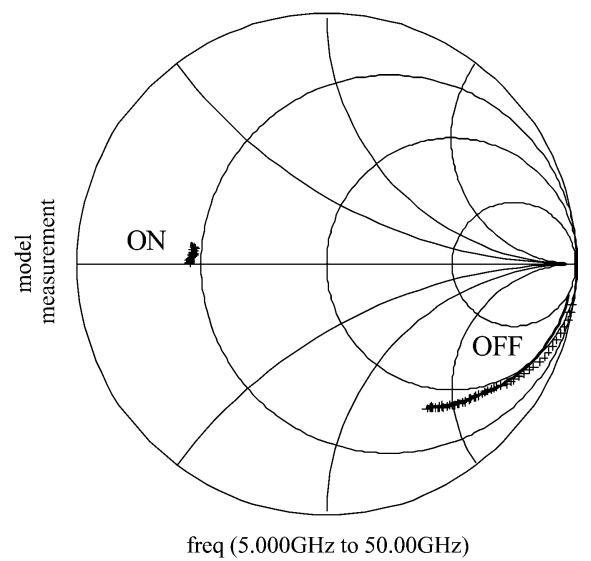

Fig. 5. Simulated and measured $S_{22} .+$ : measured results; solid line: simulated results.

size of $1 \times 1 \mathrm{~mm}^{2}$. When VP is in the high state and VN is in the low state, the phase delay of the signal path is $0^{\circ}$. Contrarily, when VP is low and VN is high, the phase delay of the signal path is $180^{\circ}$. The amplitude modulation can also be performed by adjusting the control voltages of VP and VN differentially, but the linearity is worse than that for diode-based mixers [1]-[4]. 


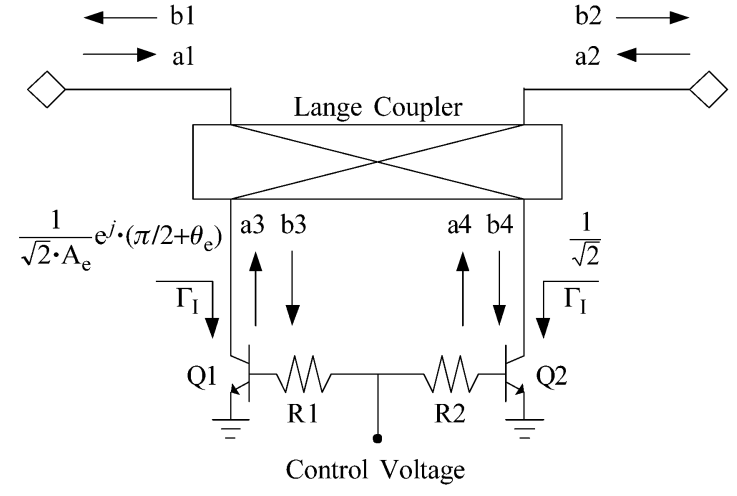

Fig. 6. Schematic of the reflection modulator with phase imbalance and amplitude imbalance.

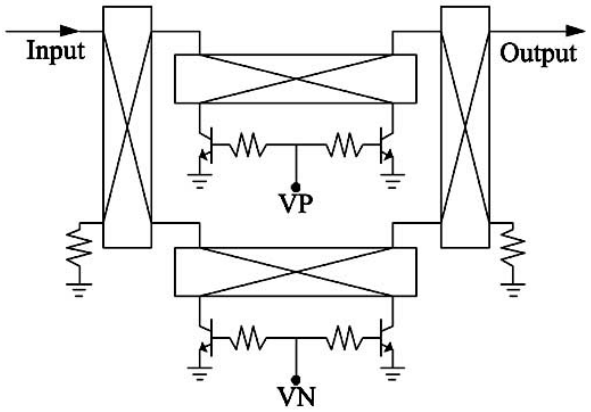

(a)

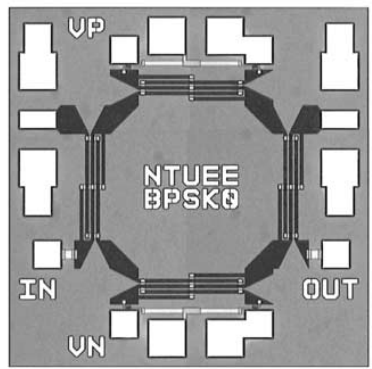

(b)

Fig. 7. (a) Schematic diagram of the BPSK modulator. (b) Microphotograph of the BPSK MMIC with a chip size of $1 \times 1 \mathrm{~mm}^{2}$.

For BPSK modulation, there are two phase states $\left(0^{\circ}\right.$ and $\left.180^{\circ}\right)$ with the same amplitude, where the two states are represented as state-0 (VP is low and VN is high) and state-1 (VP is high and VN is low). The HBT devices were operated in the ON state when the control voltage is high and the OFF state when the control is low. From (7), the transmission coefficient $S_{21}$ of the BPSK modulator can be expressed as

$$
S_{21}=\frac{1}{2}\left[\frac{\Gamma_{I}}{A_{e}^{3}} e^{j\left(3 \pi / 2+3 \cdot \theta_{e}\right)}+\frac{\bar{\Gamma}_{I}}{A_{e}} e^{j\left(\pi / 2+\theta_{e}\right)}\right]
$$

where $\bar{\Gamma}_{I}$ is the input reflection coefficient of the HBT at VN port and $\Gamma_{I}$ is the input reflection coefficient of the HBT at VP port. Let us assume that an amplitude imbalance of $3 \mathrm{~dB}$ and a phase imbalance of $5^{\circ}$ are included in Lange coupler. From (8), it can be observed that, when the magnitudes of the ON- and OFF-state reflection coefficients are equal to 1 at $180^{\circ}$ out of phase, the insertion loss and the imbalance will be minimized. However, if $\Gamma_{I}$ and $\bar{\Gamma}_{I}$ were not perfectly balanced, the impact to the circuit performance can be investigated as follows. The magnitude difference of the reflection coefficient between $\Gamma_{I}$ and $\bar{\Gamma}_{I}$ was swept from 0 to 1 , the insertion losses of two states were plotted in Fig. 8(a), and the amplitude and phase imbalances were plotted in Fig. 8(b). We can see that the insertion losses and the imbalances (amplitude and phase) are degraded with the magnitude difference increasing. The phase difference between $\Gamma_{I}$ and $\bar{\Gamma}_{I}$ is swept from $0^{\circ}$ to $180^{\circ}$, the insertion losses of two states are plotted in Fig. 8(c), and the amplitude and phase imbalances are plotted in Fig. 8(d). Also, the degradation of insertion loss and imbalance increases with the phase difference.

From the discussion above, we can see that the performance of the BPSK modulator is dominated by the reflection coefficient of $\Gamma_{I}$ and $\bar{\Gamma}_{I}$. It shows the importance of proper selection of appropriate impedance for the ON and OFF states of the cold HBTs, especially for a broad-band design. To minimize the insertion losses and amplitude and phase imbalance, the magnitude of the ON- and OFF-state reflection coefficients must be close to 1 and $180^{\circ}$ out of phase. The BPSK modulator was implemented with microstrip lines. The Lange couplers and transmission lines are simulated with a full-wave electromagnetic (EM) simulator [22], and then the results are used with the HBT model presented in Section II to simulate the performance of the BPSK modulator. The simulation results show an insertion loss of $6 \mathrm{~dB}$, an amplitude imbalance of $1 \mathrm{~dB}$, and a phase imbalance of $5^{\circ}$ between $50-100 \mathrm{GHz}$. The simulated input/output return losses are better than $10 \mathrm{~dB}$.

\section{B. IQ Modulator}

The schematic of the IQ modulator is shown in Fig. 9, which consists of two BPSK modulators, a Lange coupler, and a Wilkinson power combiner. The BPSK modulator is based on a balanced reflection-type phase shifter, which features low insertion loss, broad-band, and good amplitude and phase balance. In Fig. 9, IP and IN are the baseband input ports of the in-phase BPSK modulator, while QP and QN are the baseband input ports of a quadrature-phase BPSK modulator. The vector summation of in-phase and quadrature-phase BPSK modulators can be in many phase and amplitude states by adjusting the control voltages (IP, IN, QP, and QN). The microphotograph of the IQ modulator MMIC was shown in [14] with a chip size of $2 \times 2 \mathrm{~mm}^{2}$. For quadrature phase-shift keying (QPSK) modulation, there are four phase states with the same amplitude, which are represented as states of $(0,0),(0,1),(1,0)$, and $(1,1)$. Assuming that the Wilkinson combiner has a phase imbalance of $\theta_{e w}$ and an amplitude imbalance of $A_{e w}$, the transmission coefficient $S_{21}$ of the modulator can be expressed as

$$
\begin{aligned}
S_{21}=\frac{1}{4}\left\{\frac{e^{j\left(\theta_{e w}\right)}}{A_{e w}}\left[\frac{\Gamma_{I}}{A_{e}^{4}} e^{j\left(4 \cdot \theta_{e}\right)}+\frac{\bar{\Gamma}_{I}}{A_{e}^{2}} e^{j\left(\pi+2 \cdot \theta_{e}\right)}\right]\right. \\
\left.+\left[\frac{\Gamma_{Q}}{A_{e}^{3}} e^{j\left(3 \pi / 2+3 \cdot \theta_{e}\right)}+\frac{\bar{\Gamma}_{Q}}{A_{e}} e^{j\left(\pi / 2+\theta_{e}\right)}\right]\right\}
\end{aligned}
$$

where $\Gamma_{I}$ and $\overline{\Gamma_{I}}$ are the input reflection coefficients of the HBT for the I-channel BPSK modulator, while $\Gamma_{Q}$ and $\bar{\Gamma}_{Q}$ are for $Q$-channel BPSK modulator. 


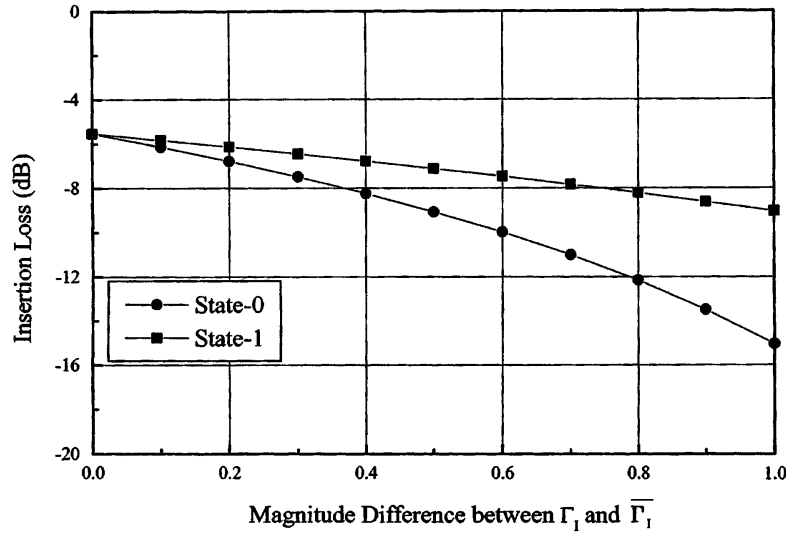

(a)

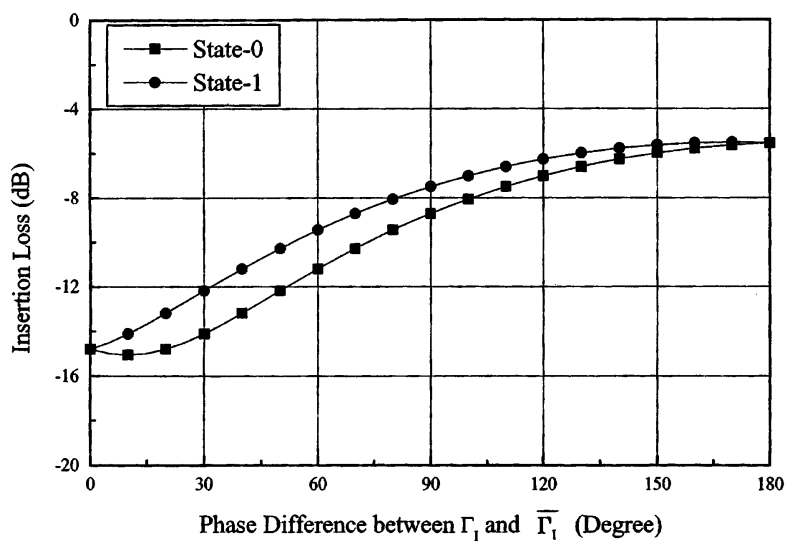

(c)

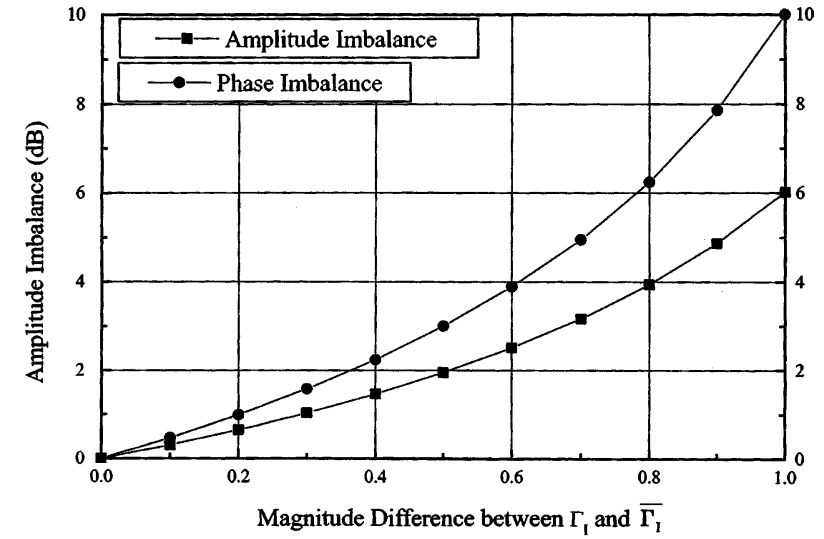

(b)

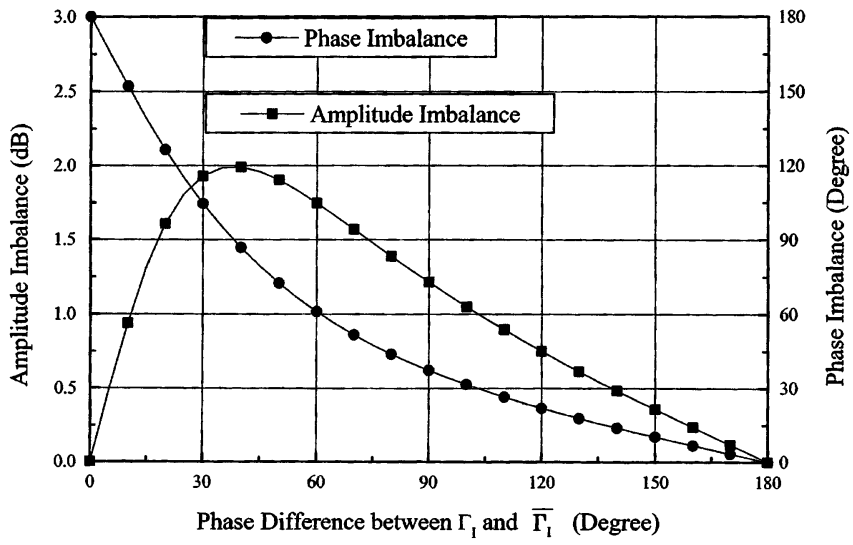

(d)

Fig. 8. With an amplitude imbalance of $3 \mathrm{~dB}$ and a phase imbalance of $5^{\circ}$, the following scenarios are plotted. (a) Insertion losses of two states versus magnitude difference of reflection coefficient swept from 0 to 1. (b) Amplitude imbalance and phase imbalance versus magnitude difference of reflection coefficient swept from 0 to 1 . (c) Insertion losses of two states versus phase difference of reflection coefficient swept from $0^{\circ}$ to $180^{\circ}$. (d) Amplitude imbalance and phase imbalance versus phase difference of reflection coefficient swept from $0^{\circ}$ to $180^{\circ}$.

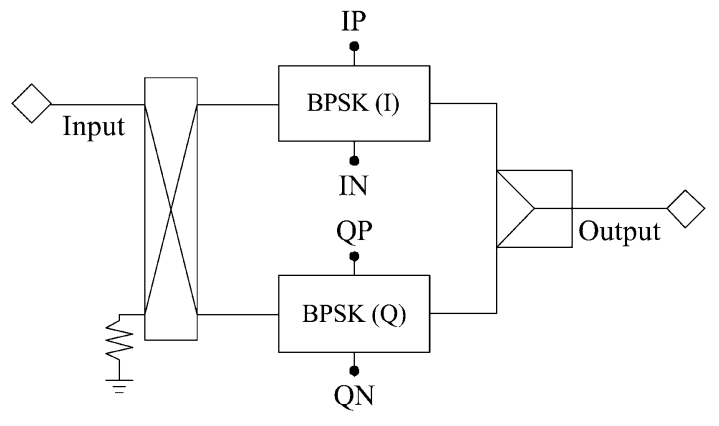

Fig. 9. Schematic diagram of the IQ modulator.

From (9), when $\Gamma_{I}\left(\Gamma_{Q}\right)$ and $\overline{\Gamma_{I}}\left(\overline{\Gamma_{Q}}\right)$ are perfectly balanced, the insertion loss of the modulator will be minimum and the imbalance of four states will be zero. If $\Gamma_{I}\left(\Gamma_{Q}\right)$ and $\overline{\Gamma_{I}}\left(\overline{\Gamma_{Q}}\right)$ are not perfectly balanced, the impact to the circuit performance can also be investigated as follows. First, suppose that phase imbalance $\left(\theta_{\text {ew }}\right)$ and amplitude imbalance $\left(A_{e w}\right)$ occur only at the Wilkinson power combiner. The phase imbalance $\left(\theta_{\text {ew }}\right)$ and the amplitude imbalance $\left(A_{e w}\right)$ represent the phase and amplitude differences between two input ports, respectively. The amplitude imbalance $\left(A_{e w}\right)$ of the Wilkinson combiner is swept from 0 to $3 \mathrm{~dB}$ and the constellation diagram of the IQ modulator is plotted in Fig. 10(a), which shows that the IQ amplitude imbalance increases with $A_{e w}$; the phase imbalance $\left(\theta_{e w}\right)$ of the
Wilkinson combiner is swept from $0^{\circ}$ to $45^{\circ}$, and the constellation diagram is plotted in Fig. 10(b), which shows the quadrature error increasing with $\theta_{e w}$. However, the phase and amplitude imbalances of the Wilkinson power combiner are very small in this design due to its symmetry. Second, suppose that phase imbalance $\left(\theta_{e}\right)$ and amplitude imbalance $\left(A_{e}\right)$ occur only at the Lange coupler. The amplitude imbalance $\left(A_{e}\right)$ is swept from 0 to $3 \mathrm{~dB}$ and the results are plotted in Fig. 10(c), which shows insertion loss and IQ amplitude imbalance increasing with $A_{e}$; the phase imbalance $\left(\theta_{e}\right)$ is swept from $0^{\circ}$ to $45^{\circ}$ and the results are plotted in Fig. 10(d), which shows quadrature error increasing with $\theta_{e}$. By a full-wave EM simulator [22], the phase imbalance of the Lange coupler is small and below $5^{\circ}$ for a general case, however, the amplitude imbalance is in the range of $0-3 \mathrm{~dB}$ based on the coupling coefficient and bandwidth requirement. The insertion loss and IQ imbalance of the IQ modulator will be influenced by the imbalance of the Lange coupler. Finally, consider the characteristics of the HBT device: the magnitude difference $\left(A_{\Gamma}\right)$ between $\Gamma_{I}\left(\Gamma_{Q}\right)$ and $\overline{\Gamma_{I}}\left(\bar{\Gamma}_{Q}\right)$ was swept from 0 to 1, the phase difference $\left(\theta_{\Gamma}\right)$ between $\Gamma_{I}\left(\Gamma_{Q}\right)$ and $\bar{\Gamma}_{I}\left(\bar{\Gamma}_{Q}\right)$ was swept from $180^{\circ}$ to $0^{\circ}$, and the results are plotted in Fig. 10(e) and Fig. 10(f), which shows insertion loss increasing with the magnitude difference. From this analysis, it is observed that the insertion loss of the IQ modulator is dominated by the phase difference and amplitude difference between $\Gamma_{I}\left(\Gamma_{Q}\right)$ and $\overline{\Gamma_{I}}\left(\bar{\Gamma}_{Q}\right)$. 

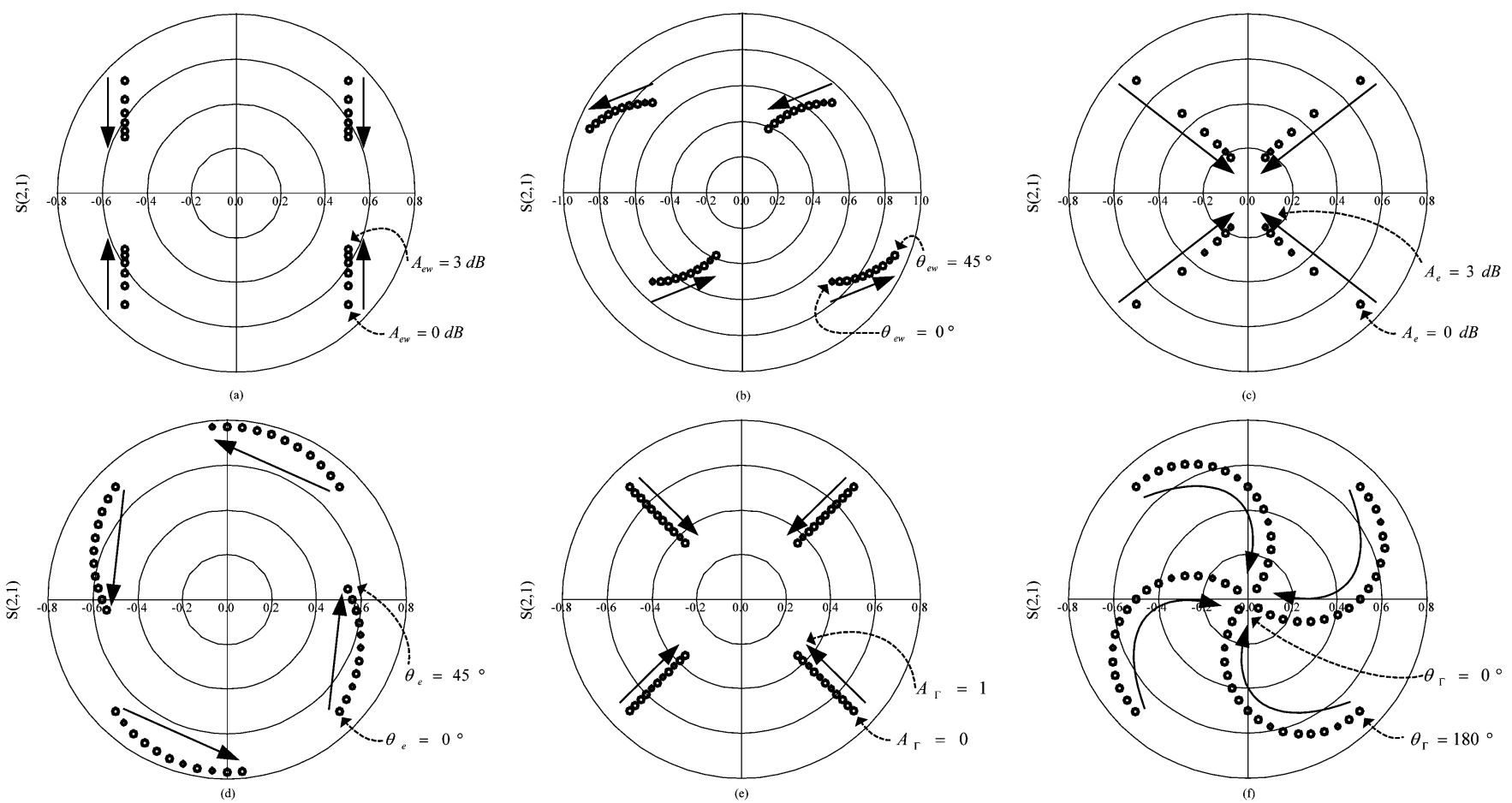

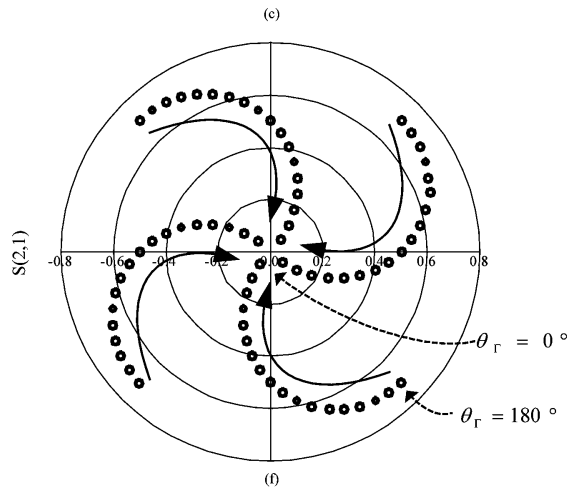

Fig. 10. Constellation diagram of the IQ modulator versus imbalance. (a) Amplitude imbalance $\left(A_{e w}\right)$ of the Wilkinson combiner was swept from 0 to $3 \mathrm{~dB}$, and IQ amplitude imbalance occurred. (b) Phase imbalance $\left(\theta_{\text {ew }}\right)$ of the Wilkinson combiner was swept from $0^{\circ}$ to $45^{\circ}$, and quadrature error occurred. (c) Amplitude imbalance $\left(A_{e}\right)$ of the Lange coupler was swept from 0 to $3 \mathrm{~dB}$, insertion loss increased, and IQ amplitude imbalance occurred. (d) Phase imbalance $\left(\theta_{e}\right)$ of the Lange coupler was swept from $0^{\circ}$ to $45^{\circ}$, and quadrature error occurred. (e) Magnitude difference $\left(A_{\Gamma}\right)$ between $\Gamma_{I}\left(\Gamma_{Q}\right)$ and $\bar{\Gamma}_{I}\left(\bar{\Gamma}_{Q}\right)$ was swept from 0 to 1 , and insertion loss increased. (f) Phase difference $\left(\theta_{\Gamma}\right)$ between $\Gamma_{I}\left(\Gamma_{Q}\right)$ and $\bar{\Gamma}_{I}\left(\bar{\Gamma}_{Q}\right)$ was swept from $180^{\circ}$ to $0^{\circ}$, and insertion loss increased.

For minimum insertion loss of the IQ modulator, the amplitude and phase imbalance of the Lange coupler and Wilkinson power combiner should be minimized. Also, the magnitude of the ON- and OFF-state reflection coefficients must be close to 1 with $180^{\circ}$ out of phase. In our IQ modulator circuit design, we used the HBT model described in Section II for the circuit simulation. The transmission lines, Lange couplers, Wilkinson power combiners, and thin-film resistors are also simulated with a full-wave EM simulator [22]. The simulated insertion losses of four states are better than $10 \mathrm{~dB}$, which feature an amplitude imbalance of within $2 \mathrm{~dB}$ and a phase imbalance of within $5^{\circ}$ between 50-100 GHz. The simulated input and output return losses are better than $10 \mathrm{~dB}$ between $50-110 \mathrm{GHz}$.

\section{EXPERIMENTAL RESULTS AND CHARACTERIZATION}

\section{A. S-Parameters' Measurement}

The $S$-parameters' measurements in the $10-110-\mathrm{GHz}$ frequency range were performed using an HP8510 MMW network analyzer and a Cascade probe station. The simulated and measured insertion losses of two states from 10 to $110 \mathrm{GHz}$ for the BPSK modulator are plotted in Fig. 11, which features the insertion losses of less than $8 \mathrm{~dB}$ between $45-95 \mathrm{GHz}$. The measurement results and simulation results have good agreement below $100 \mathrm{GHz}$. The amplitude imbalance is within $2.5 \mathrm{~dB}$ and the phase imbalance is within $5^{\circ}$ between $45-90 \mathrm{GHz}$. However, the measured insertion losses of the zero state were degraded for $100 \mathrm{GHz}$ and above because the HBT bias conditions for the VP port and $\mathrm{VN}$ port are different. The HBT at the $\mathrm{VN}$ port is biased under the open collector condition, while at VP port it is biased with $50 \Omega$ at the collector. The other reason for the degraded

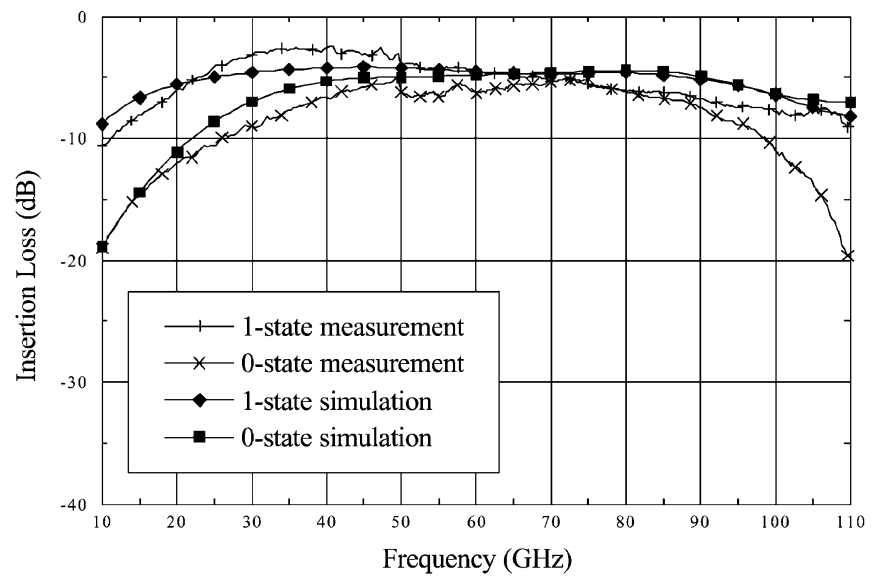

Fig. 11. Simulated and measured insertion losses of two states from 10 to $110 \mathrm{GHz}$ for the BPSK modulator.

insertion loss is that the magnitude difference of HBT reflection coefficients between the ON and OFF states becomes large for high-frequency operation (above $100 \mathrm{GHz}$ ), as discussed in Fig. 7(a). To minimize the imbalance of the modulator, the device bias conditions at the $\mathrm{VP}$ and $\mathrm{VN}$ ports should be the same. We need to add some dc-block capacitors between the $50-\Omega$ termination resistor and the Lange coupler or the HBT's collector and the Lange coupler. However, this will degrade circuit performance at low frequencies. From 50 to $100 \mathrm{GHz}$, the worst-case input/output return losses are better than $10 \mathrm{~dB}$ due to the balanced circuit architecture in the BPSK modulator.

Good amplitude and phase match of the BPSK is achieved between 70-85 GHz, with an amplitude imbalance below $1 \mathrm{~dB}$ and phase imbalance below $1^{\circ}$. To minimize the imbalance of 


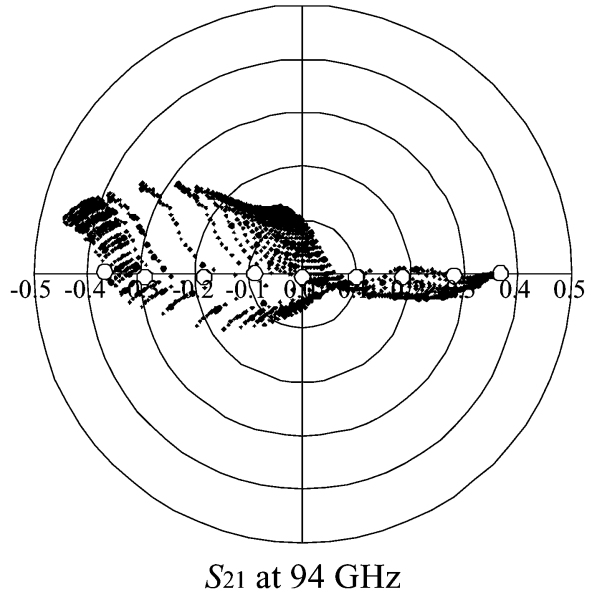

Fig. 12. Measured static constellation diagram of the HBT BPSK modulator at $94 \mathrm{GHz}$ (measured $S$-parameters: •; extracted BPSK and multilevel ASK: $\bigcirc)$.

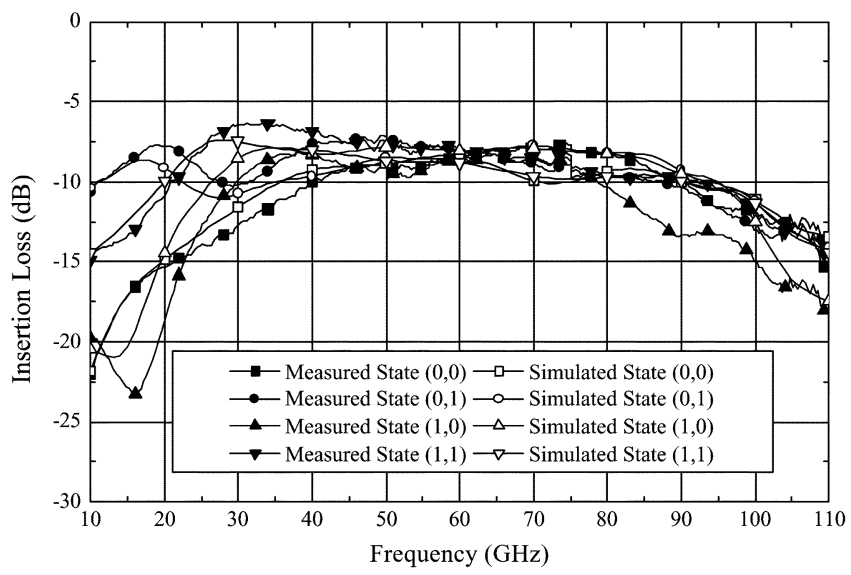

Fig. 13. Simulated and measured insertion losses of two states from 10 to $110 \mathrm{GHz}$ for the IQ modulator.

the BPSK modulator for other frequencies, we can use the static constellation diagram measurement [12] to extract the best phase and amplitude states. The static constellation diagrams were obtained from continuous-wave $(\mathrm{CW})$ mode $S$-parameters' measurement with a computer-controlled setup. The forward transmission coefficients $S_{21}$ are plotted in Fig. 12 with a linear polar format, where the control voltages of VP and VN have been swept from 0 to $4 \mathrm{~V}$ in steps of $0.2 \mathrm{~V}$. Based on the forward transmission coefficients, EVM calculation is used to extract the best amplitude and phase states. We generate BPSK and multilevel amplitude shift keying (ASK) as an example, and the results are also plotted in Fig. 12 with a symbol of "o," which features a minimum insertion loss of $8 \mathrm{~dB}$, an amplitude imbalance of within $0.5 \mathrm{~dB}$, and a phase imbalance of within $0.5^{\circ}$. The static constellation diagram at 50 and $110 \mathrm{GHz}$ were also performed, and the results are similar to those shown in Fig. 12. For ultrawide-band applications, the amplitude imbalance and phase imbalance can be further improved this way.

For the IQ modulator MMIC, the simulated and measured insertion losses of the four states from 10 to $110 \mathrm{GHz}$ are plotted in Fig. 13. The insertion losses of the four states are below $12.5 \mathrm{~dB}$, the amplitude imbalance is within $2.5 \mathrm{~dB}$, and the phase imbalance is within $15^{\circ}$ between $50-85 \mathrm{GHz}$. From 60 to $75 \mathrm{GHz}$, the
$S$-parameters of this MMIC demonstrate good phase and amplitude match, which feature an insertion loss of $8 \mathrm{~dB}$, amplitude imbalance of $1 \mathrm{~dB}$, and phase imbalance of $2^{\circ}$. The measured and simulated results are in good agreement below $80 \mathrm{GHz}$. The insertion loss of the measured state $(1,0)$ are degraded above $80 \mathrm{GHz}$, and this is because the HBT bias condition (open collector) at the QN port is different from the other ports (biased with $50 \Omega$ at the collector). The other reason for the degraded insertion loss is that the magnitude difference of HBT reflection coefficients between the ON and OFF states becomes large for high-frequency operation, as discussed in Fig. 10(e). The simulated and measured input and output return losses are better than $8 \mathrm{~dB}$ between $50-100 \mathrm{GHz}$. The bandwidth of the input return loss is wider than the output return loss due to the balanced architecture used in the input port.

If we apply a high-level modulation format (n-QAM, n-PSK) to the IQ modulator directly, the amplitude and phase states will be distorted. This is because the relationship between the magnitude of the HBT reflection coefficient and base control voltage is nonlinear. Therefore, we use the calibration bias method [12] via the static constellation diagram measurement to extract exact amplitude/phase states and minimize the amplitude imbalance and phase imbalance of the IQ modulator. The forward transmission coefficients at $94 \mathrm{GHz}$ are reported in [14]. Based on forward transmission coefficients, EVM calculation is also used to extract the best amplitude and phase states. We can generate the static constellation diagrams of QPSK or higher order QAM modulations. The 64-QAM extracted constellation diagram can also be seen in [14]. The extraction of the QPSK format at 50 and $110 \mathrm{GHz}$ were also performed, with results similar to those in [14] with slightly different control voltages. For broad-band operation, the lookup table (LUT) method [23] can be used to deal with the different control voltages at IP, IN, QP, and QN ports.

\section{B. Spectrum Characterization}

In the case of the BPSK modulator, we assume that the baseband signal between the VP and VN ports is $\cos \left(\omega_{B} t\right)$, and the carrier signal used for the modulator is $\cos \left(\omega_{\mathrm{LO}} t\right)$, where $\omega_{B}$ is expressed for the angle frequency of the baseband signal and $\omega_{\mathrm{LO}}$ is expressed for the angle frequency of the carrier signal. Under baseband signal excitation at the base, the collector-emitter impedance can be expressed as a Taylor series with the base voltage $V_{b}$ [24], [25]. Therefore, the reflection coefficient of the HBT can also be expressed as a power series in terms of $\cos \left(\omega_{B} t\right)$. For simplicity, a time-varying output voltage of the BPSK modulator can be expressed as

$$
\begin{aligned}
V_{\mathrm{out}}^{\mathrm{BPSK}}(t)=-\frac{j}{2}\left[C_{0}\right. & +C_{1} \cos \left(\omega_{B} t\right)+C_{2} \cos ^{2}\left(\omega_{B} t\right) \\
& \left.+C_{3} \cos ^{3}\left(\omega_{B} t\right)+\cdots\right] \cos \left(\omega_{\mathrm{LO}} t\right)
\end{aligned}
$$

where $C_{0}$ is the dc term, which causes LO leakage and dc offset effect, $C_{1}$ is the first-order term and is proportional to the conversion gain (loss) of the modulator, $C_{2}, C_{4}, C_{6}, \ldots$, are the even-order terms that cause even harmonics, and $C_{3}, C_{5}, C_{7}, \ldots$, are the odd-order terms that cause odd harmonics and adjacent channel spurious. 


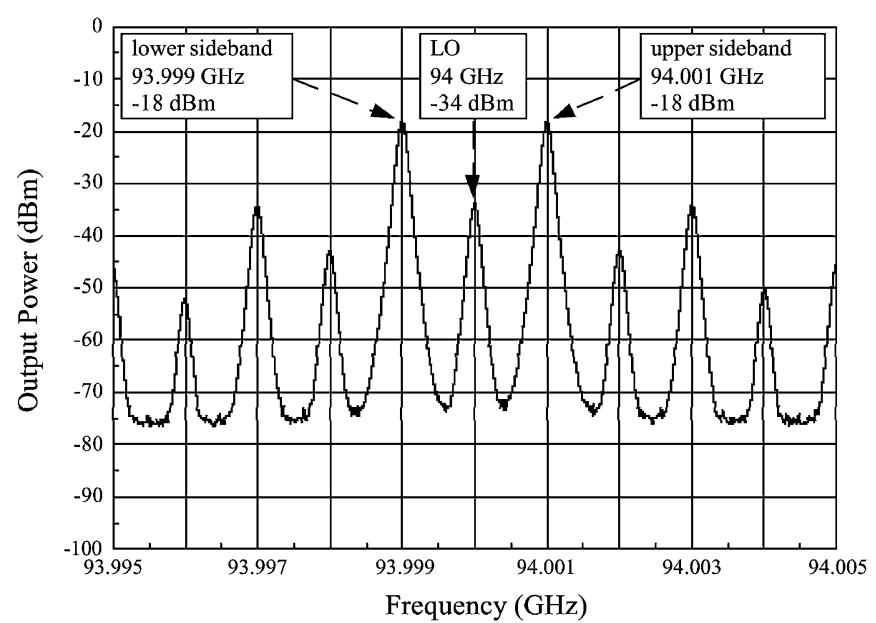

Fig. 14. Measured output spectrum of the BPSK modulator. The LO frequency is $94 \mathrm{GHz}$ and the IF frequency is $1 \mathrm{MHz}$.

For optimal operation, the LO leakage (dc offset) should be minimized since an unwanted carrier signal at the RF output can degrade the modulation quality of the high-level amplification. DC offset compensation techniques have been proposed in the literature, including a compensation scheme and operation principle [26], [27]. By using these techniques, the LO leakage problem can be improved substantially. The conversion loss of the modulator is dominated by $C_{1}$. Consequently, $C_{1}$ should be maximized in order to minimize the conversion loss. The higher order terms (even and odd) affect the linearity of the modulator, which are caused by the characteristic of the HBT device in this paper.

To evaluate the LO leakage and spurious suppression for the BPSK modulator, we used two CW sources (one is for the LO, the other is for baseband) and a spectrum analyzer. The baseband signal is a 1-MHz sinusoidal waveform with $2-V_{p-p}$ amplitude and the dc bias for the VP and VN ports is $2 \mathrm{~V}$. The measured output spectrum of the BPSK modulator as a double-sideband up-converter is plotted in Fig. 14. The LO frequency is $94 \mathrm{GHz}$ with a power level of $-8 \mathrm{dBm}$. As can be observed, the upper sideband signal $(94.001 \mathrm{GHz})$ and lower sideband signal $(93.999 \mathrm{GHz})$ are about $-18 \mathrm{dBm}$ and the LO leakage power is about $-34 \mathrm{dBm}$. Therefore, the LO suppression of the modulator is greater than $16 \mathrm{~dB}$. Similarly, evaluations were also performed at 50,60, 77, and $110 \mathrm{GHz}$. Only at $110 \mathrm{GHz}$ should the dc offset compensation be applied to the modulator, and then the suppression can be improved up to $50 \mathrm{~dB}$.

For the IQ modulator, we assume that the baseband signal between the IP and IN ports is $\cos \left(\omega_{B} t\right)$; the baseband signal between the QP and QN ports is $-\sin \left(\omega_{B} t\right)$, and the carrier signal used for the modulator is $\cos \left(\omega_{\mathrm{LO}} t\right)$. By (9) and (10), a time-varying output voltage of the IQ modulator can be calculated as

$$
\begin{aligned}
V_{\mathrm{out}}^{\mathrm{IQ}}=\frac{1}{4}[ & \frac{\cos \left(\omega_{\mathrm{LO}} t+\theta_{e w}+\theta_{e}\right)}{A_{e w} A_{e}} \\
& \cdot\left(C_{0}+C_{1} \cos \left(\omega_{B} t\right)+C_{2} \cos ^{2}\left(\omega_{B} t\right)\right. \\
& \left.+C_{3} \cos ^{3}\left(\omega_{B} t\right)+\cdots\right)-\sin \left(\omega_{\mathrm{LO}} t\right) \\
& \cdot\left(-C_{0}+C_{1} \sin \left(\omega_{B} t\right)+C_{2} \sin ^{2}\left(\omega_{B} t\right)\right. \\
& \left.\left.+C_{3} \sin ^{3}\left(\omega_{B} t\right)+\cdots\right)\right]
\end{aligned}
$$

where the coefficients of $C_{0}, C_{1}, C_{2}, \cdots$ are the same with (10). However, the LO leakage term can be expressed as

$$
V_{\mathrm{LO}}^{\mathrm{IQ}}=\frac{1}{4}\left[\frac{\cos \left(\omega_{\mathrm{LO}} t+\theta_{e w}+\theta_{e}\right)}{A_{e w} A_{e}}+\sin \left(\omega_{\mathrm{LO}} t\right)\right] C_{0} .
$$

From (12), the LO magnitude can be expressed as

$$
\mathrm{LO}_{\text {mag }}=\frac{1}{4} C_{0} \sqrt{\left(\frac{1}{A_{e w} A_{e}}\right)^{2}-2 \frac{1}{A_{e w} A_{e}} \sin \left(\theta_{e w}+\theta_{e}\right)+1} .
$$

The first-order term can then be expressed as

$$
\begin{aligned}
V_{\text {First-order }}^{\mathrm{IQ}}=\frac{1}{4} C_{1}[ & \frac{1}{2 A_{e w} A_{e}} \cos \left(\omega_{\mathrm{LO}} t+\omega_{B} t+\theta_{e w}+\theta_{e}\right) \\
+ & \frac{1}{2} \cos \left(\omega_{\mathrm{LO}} t+\omega_{B} t\right)+\frac{1}{2 A_{e w} A_{e}} \\
& \cdot \cos \left(\omega_{\mathrm{LO}} t-\omega_{B} t+\theta_{e w}+\theta_{e}\right) \\
& \left.\cdot-\frac{1}{2} \cos \left(\omega_{\mathrm{LO}} t+\omega_{B} t\right)\right]
\end{aligned}
$$

We can see that (14) includes the upper and lower sideband signals, and the magnitude of these signals can be written as follows:

$$
\begin{aligned}
& \mathrm{USB}_{\mathrm{mag}} \\
& \quad=\frac{1}{8} C_{1} \sqrt{\left(\frac{1}{A_{e w} A_{e}}\right)^{2}+2\left(\frac{1}{A_{e w} A_{e}}\right) \cos \left(\theta_{e w}+\theta_{e}\right)+1} \\
& \mathrm{LSB}_{\mathrm{mag}} \\
& =\frac{1}{8} C_{1} \sqrt{\left(\frac{1}{A_{e w} A_{e}}\right)^{2}-2\left(\frac{1}{A_{e w} A_{e}}\right) \cos \left(\theta_{e w}+\theta_{e}\right)+1 .}
\end{aligned}
$$

Through (13) and (15), the carrier suppression and sideband suppression can be evaluated as given in (16) and (17), shown at the bottom of the following page. The carrier suppression [see (16)] is a function of the coefficient $C_{0}, C_{1}$, phase imbalance, and amplitude imbalance. For low imbalance conditions (phase imbalance $<5^{\circ}$, amplitude imbalance $<3 \mathrm{~dB}$ ), the suppression is dominated by the coefficients $C_{0}$ and $C_{1}$. The coefficient $C_{0}$ is related to the device characteristic and varies with the base bias, and $C_{1}$ is related to the conversion loss of the IQ modulator (or insertion loss when the modulator is operated as a phase shifter). Similarly, the dc offset compensation can be implemented by adjusting the dc bias current at the I and Q channels. From (17), we can see that the sideband suppression is degraded by the increase of phase and amplitude imbalance. For example, assuming that the Lange coupler and Wilkinson power combiner contain a phase imbalance of $5^{\circ}$ and an amplitude imbalance of $3 \mathrm{~dB}$, the sideband suppression is approximately $9.3 \mathrm{~dB}$. Contrarily, without any imbalance, the lower sideband power level is zero. The harmonic and the adjacent channel spurious effect are caused by the high-order term $C_{2}, C_{3}, C_{4} \ldots$, which is related to device and bias conditions. Also, the high-order terms worsen the modulation quality of the IQ modulator. These nonlinear effects can be monitored with the output spectrum, and the power level of the spurious and the harmonic are proportional the high-order term. Therefore, we can evaluate the characteristic of this IQ modulator via the spectrum characterization as the measurement of the traditional IQ modulator [5]-[9]. 


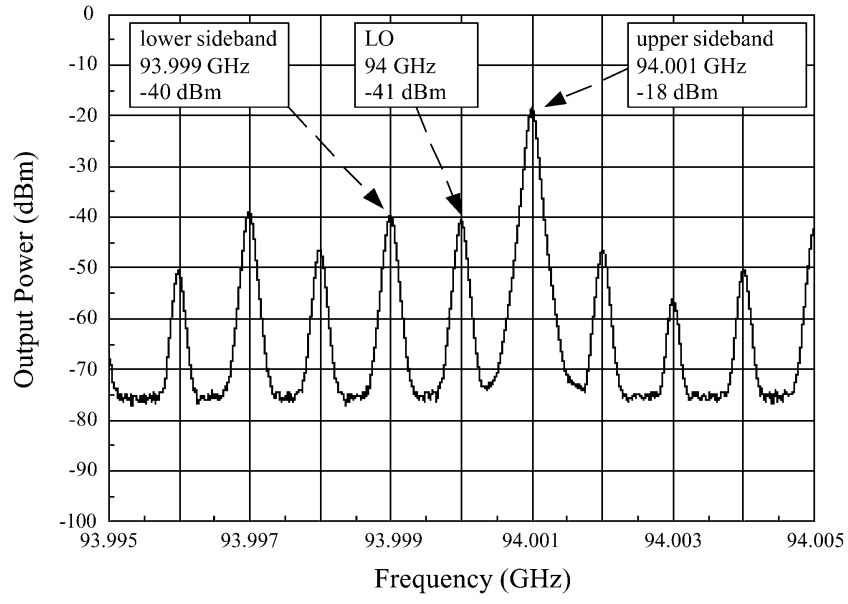

Fig. 15. Measured output spectrum of the HBT IQ modulator as an image rejection up-converter. The LO frequency is $94 \mathrm{GHz}$, the IF frequency is $1 \mathrm{MHz}$, the upper sideband RF frequency is $94.001 \mathrm{GHz}$, and the lower sideband RF frequency is $93.999 \mathrm{GHz}$.

Spectrum characterization for the IQ modulator was performed as an image rejection up-converter. We used two signal generators for the baseband (or IF) inputs at the I and Q channels, which are 1-MHz sinusoid with amplitude of $2-V_{p-p}$ and the phase differenve between I and Q is $90^{\circ}$. An MMW $\mathrm{CW}$ source was used for the $\mathrm{LO}$ with a power level of $-8 \mathrm{dBm}$. The dc biases applied to the IP, IN, QP, and QN ports are $2 \mathrm{~V}$ for normal bias conditions. At $94 \mathrm{GHz}$, the measured output spectrum of the IQ modulator is plotted in Fig. 15, which features an LO suppression of $22 \mathrm{~dB}$, a sideband suppression of $21 \mathrm{~dB}$, and a spurious rejection of better than $21 \mathrm{~dB}$. Again, as an example, assuming that the total amplitude error $\left(A_{e w}\right.$ and $\left.A_{e}\right)$ is $1 \mathrm{~dB}$, then the total phase error $\left(\theta_{e w}\right.$ and $\left.\theta_{e}\right)$ can be carried out about $8^{\circ}$ from (17). The spectrum characterizations were also performed at 50, 60, 77, and $110 \mathrm{GHz}$. For normal bias conditions (IP, IN, QP, and QN ports were applied at 2-V dc bias), the LO suppression is not sufficient $(<15 \mathrm{~dB})$ at $110 \mathrm{GHz}$, however, we can apply the dc offset compensation to the IQ modulator and improve the LO suppression up to $50 \mathrm{~dB}$. For general QPSK modulation applications, LO suppression of greater than $20 \mathrm{~dB}$ and sideband and spurious suppression of greater than $20 \mathrm{~dB}$ are acceptable, because the QPSK signal can tolerate more phase error and amplitude error [or a low signal-to-noise ratio (SNR)] compared with the n-QAM and n-PSK modulation signals.

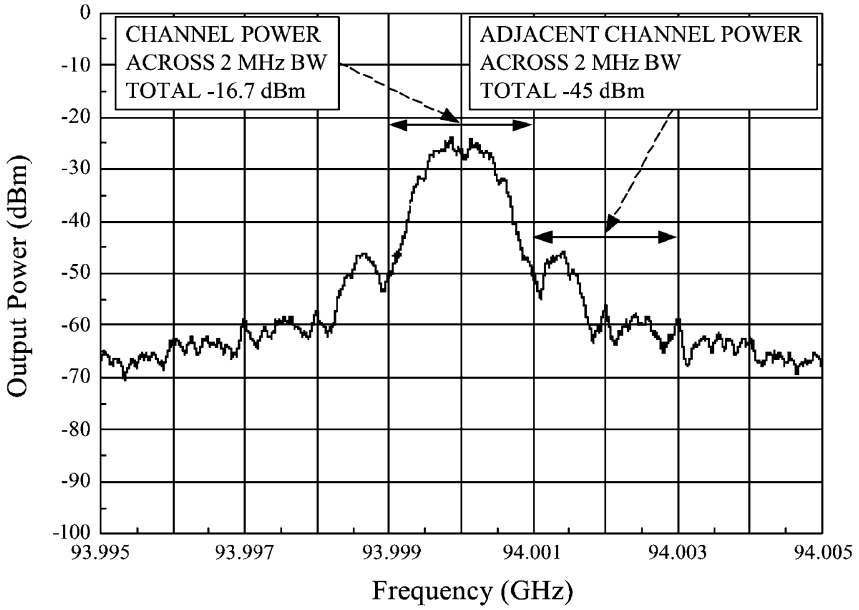

Fig. 16. Measured output spectrum of the HBT BPSK modulator. The main channel power is $-16.7 \mathrm{dBm}$ and the adjacent channel power is $-45 \mathrm{dBm}$ with a channel bandwidth of $2 \mathrm{MHz}$.

\section{Vector Signal Characterization}

For vector signal characterization, we use our recently reported vector signal measurement system for the MMW BPSK and IQ modulator measurement, which features maximum operation frequency of $110 \mathrm{GHz}$, a maximum symbol rate of $1 \mathrm{Ms} / \mathrm{s}$, a and maximum analysis bandwidth of $3 \mathrm{MHz}$ and supports multiple digital modulation formats [16].

In the setup of the vector signal characterization, the LO power is about $-8 \mathrm{dBm}$ and the amplitude of the baseband signal is $2 V_{p-p}$. To minimize the spectrum spread, we used a root raised cosine (RRC) filter with a $0.5-\alpha$ value to filter the baseband signal. The data frame is coded with a 1-Ms/s symbol rate, a pseudorandom bit stream (PRBS), and the mapping table used with the extracted results from static constellation diagram measurement. For the BPSK modulator, the baseband signal is applied to the VP and VN ports, which are coded with a BPSK modulation format. The measured modulation spectrum of the BPSK modulator is plotted in Fig. 16. Assuming that the channel spacing and channel bandwidth are both $2 \mathrm{MHz}$, therefore, the main channel power is $-16.7 \mathrm{dBm}$ and the adjacent channel power is $-45 \mathrm{dBm}$. As can be observed, the ACPR is better than $28 \mathrm{dBc}$. The measured BPSK modulation quality results at $94 \mathrm{GHz}$ are plotted in Fig. 17, including the IQ vector diagram, the eye diagram, error versus time plot and performance summary that show the measured EVM of $6.7 \%$, amplitude error of $3.9 \%$, and phase error of 3.1.

$$
\begin{aligned}
& \text { Suppression }_{\mathrm{USB}-\mathrm{LO}}=\frac{\mathrm{USB}_{\mathrm{mag}}}{L O_{\mathrm{mag}}}=10 \log \left[\frac{\frac{1}{4} C_{1}\left[\left(\frac{1}{A_{e w} A_{e}}\right)^{2}+2\left(\frac{1}{A_{e w} A_{e}}\right) \cos \left(\theta_{e w}+\theta_{e}\right)+1\right]}{C_{0}\left[\left(\frac{1}{A_{e w} A_{e}}\right)^{2}-2 \frac{1}{A_{e w} A_{e}} \sin \left(\theta_{e w}+\theta_{e}\right)+1\right]}\right] \\
& \text { Suppression }_{\mathrm{USB}-\mathrm{LSB}}=\frac{\mathrm{USB}_{\mathrm{mag}}}{\mathrm{LSB}_{\mathrm{mag}}}=10 \log \left[\frac{\left(\frac{1}{A_{e w} A_{e}}\right)^{2}+2\left(\frac{1}{A_{e w} A_{e}}\right) \cos \left(\theta_{e w}+\theta_{e}\right)+1}{\left(\frac{1}{A_{e w} A_{e}}\right)^{2}-2\left(\frac{1}{A_{e w} A_{e}}\right) \cos \left(\theta_{e w}+\theta_{e}\right)+1}\right]
\end{aligned}
$$




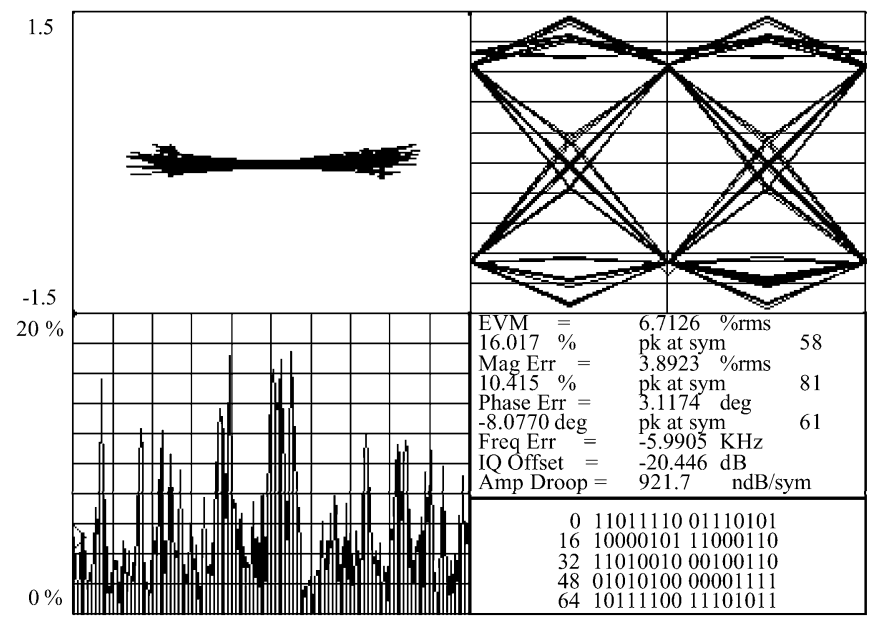

Fig. 17. Measured BPSK modulation quality results at $94 \mathrm{GHz}$, IQ vector diagram, eye diagram, error vector versus time plot, and performance summary.

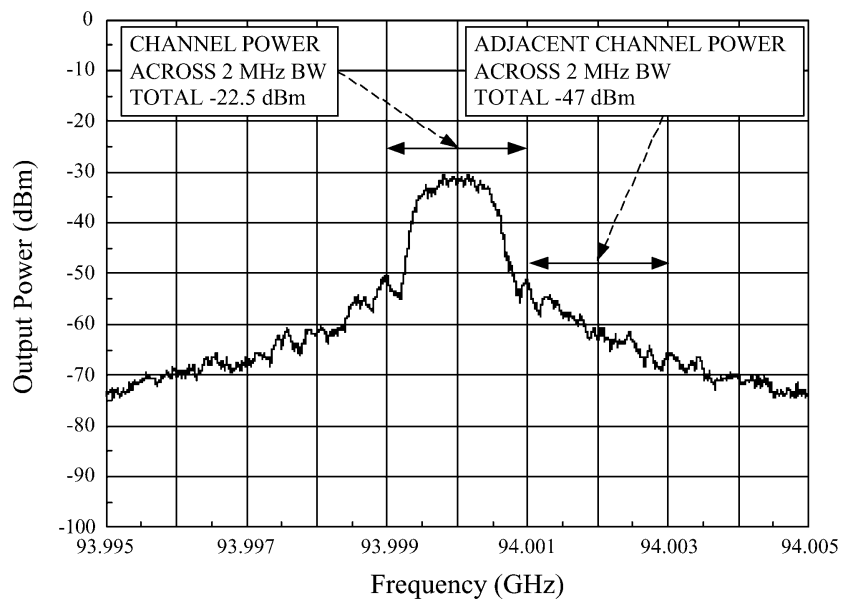

Fig. 18. Measured output spectrum of the HBT IQ modulator with 16-QAM modulationm. The output power is $-22.5 \mathrm{dBm}$ and the adjacent channel power is $-47 \mathrm{dBm}$ with a channel bandwidth of $2 \mathrm{MHz}$.

For the IQ modulator, the baseband IQ is coded with a QPSK modulation format and a 16-QAM modulation format individually. The measured output spectrum of 16-QAM modulation is plotted in Fig. 18, which shows a main channel power of $-22.5 \mathrm{dBm}$ and an adjacent channel power of $-47 \mathrm{dBm}$. Also, we can see that the ACPR is better than $-24.5 \mathrm{dBc}$ from Fig. 18 . The measured QPSK modulation quality and spectrum results at $94 \mathrm{GHz}$ were presented in [14], which features am EVM of below $9.7 \%$, an amplitude error of below $7.3 \%$ (approximately $0.65 \mathrm{~dB}$ ), and a root mean square (rms) phase error of below $3.7^{\circ}$. To compare with the calculations, we can estimate that the sideband suppression is approximately $28 \mathrm{~dB}$ from (17), which is better than the measurement results of Fig. 15 due to the calibration bias and dc offset compensation applied to baseband signal generator. It can be observed that the amplitude error has more impact than phase error, and the constellation diagram shows a quadrature error as in Fig. 10(b) and (d). Therefore, from the imbalance analysis, we found that this impact is mainly due to the phase imbalance of the coupler and the combiner. Also, if the reflection coefficients of $\Gamma_{I}\left(\Gamma_{Q}\right)$ and $\bar{\Gamma}_{I}\left(\bar{\Gamma}_{Q}\right)$ are not perfectly balanced, the quadrature error will also degrade

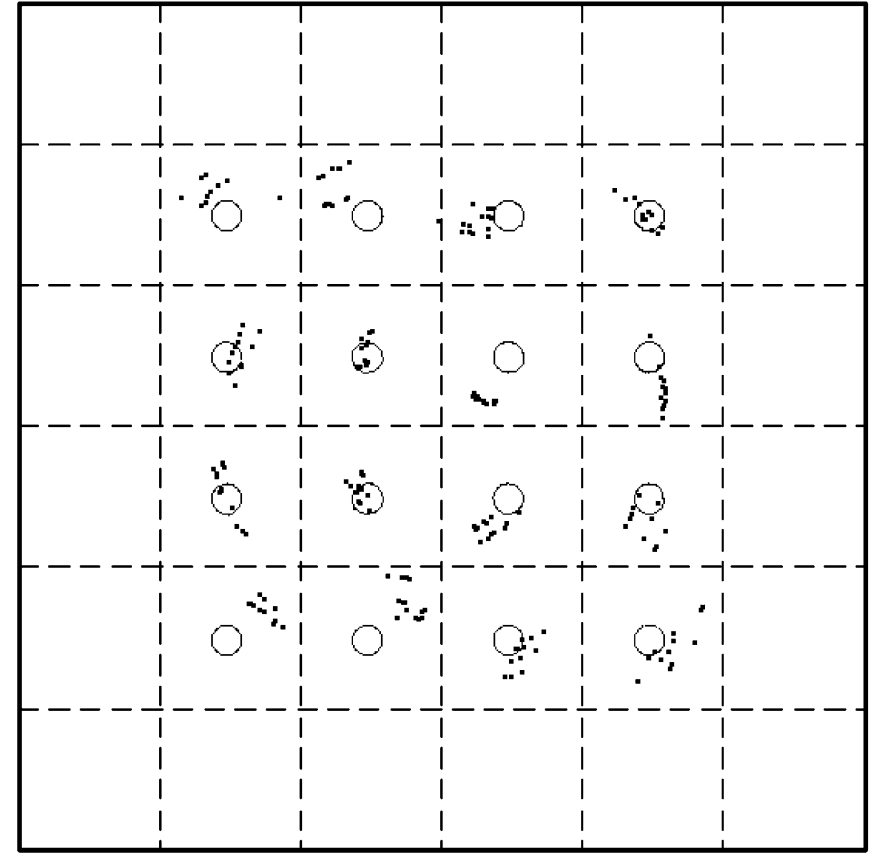

Fig. 19. Measured constellation diagram at $94 \mathrm{GHz}$ for 16-QAM modulation with a 4-Mb/s data rate (reference constellations: $\bigcirc$; recovered symbol: $\cdot$ ).

seriously. For high-level digital modulation, we apply 16-QAM modulation to the IQ modulators, and the measured constellation diagram at $94 \mathrm{GHz}$ is plotted in Fig. 19. It is observed that the recovered symbols can be clearly distinct from each other, and the performance summary features an EVM of $12 \%$, an amplitude error of $7.5 \%$, and a phase error of $8.4^{\circ}$ in rms format.

The measured EVM results for 16-QAM modulation can be explained as follows.

- From (9), the magnitude of reflection coefficients versus control voltage should be a linear relation for high-level digital modulations. Due to the nonlinearity for cold-mode devices, the linearity of reflection-type IQ modulators is usually worse than that of the diode-based IQ modulators in [2], [3].

- From the vector signal measurement results, the EVM degradation of 16-QAM modulation is worse than that of QPSK modulation because the LO phase noise requirement of 16-QAM modulation is higher. The EVM degradation is even worse for high-level digital modulations, such as n-QAM or n-PSK modulation.

Regarding the vector signal measurement, the measured EVMs in this paper also include EVM contributions from the down-converter system and the LO sources. However, we can evaluate the EVM degradation for an overall test bench with a simple THRU line. Without the baseband IQ inputs, the HP8510 $\mathrm{CW}$-mode source is like an all- 0 data-modulated signal. For the $W$-band applications, the phase noise and amplitude noise of the LO sources included from HP8510 and HP70000 may result in 3\%-5\% EVM degradation. Also, the imperfect baseband IQ sources in the test setup will also degrade the EVM performance. On the whole, the baseband IQ sources will degrade an EVM of 3\%-4\%, which can be measured by a vector signal analyzer directly. This EVM degradation of the IQ sources is due to 
TABLE III

PERFoRMANCE SUMmARY OF THE HBT BPSK AND IQ MODULATORS

\begin{tabular}{c|c|c|c|c|c|c|c|c|c|c}
\hline Frequency & \multicolumn{2}{|c|}{$50 \mathrm{GHz}$} & \multicolumn{2}{c|}{$60 \mathrm{GHz}$} & \multicolumn{2}{c|}{$77 \mathrm{GHz}$} & \multicolumn{2}{c|}{$94 \mathrm{GHz}$} & \multicolumn{2}{c}{$110 \mathrm{GHz}$} \\
\hline MMIC & BPSK & IQ & BPSK & IQ & BPSK & IQ & BPSK & IQ & BPSK & IQ \\
\hline $\begin{array}{c}\text { Insertion } \\
\text { Loss }\end{array}$ & $8 \mathrm{~dB}$ & $9 \mathrm{~dB}$ & $8 \mathrm{~dB}$ & $8 \mathrm{~dB}$ & $8 \mathrm{~dB}$ & $9 \mathrm{~dB}$ & $8 \mathrm{~dB}$ & $14 \mathrm{~dB}$ & $20 \mathrm{~dB}$ & $18 \mathrm{~dB}$ \\
\hline EVM\# & $<6 \% *$ & $<7 \% *$ & $<6 \%$ & $<12 \%$ & $<7 \%$ & $<12 \%$ & $<7 \%$ & $<10 \%$ & $<8 \%$ & $<12 \%$ \\
\hline $\begin{array}{c}\text { LO } \\
\text { Rejection }\end{array}$ & $>20 \mathrm{~dB}$ & $>21 \mathrm{~dB}$ & $>23 \mathrm{~dB}$ & $>23 \mathrm{~dB}$ & $>25 \mathrm{~dB}$ & $>21 \mathrm{~dB}$ & $>20 \mathrm{~dB}$ & $>20 \mathrm{~dB}$ & $>15 \mathrm{~dB}$ & $>15 \mathrm{~dB}$ \\
\hline $\begin{array}{c}\text { ACPR } \\
(\mathrm{dBc})\end{array}$ & $<-28$ & $<-27$ & $<-27$ & $<-26$ & $<-26$ & $<-23$ & $<-28$ & $<-24.5$ & $<-23$ & $<-21$ \\
\hline
\end{tabular}

*With calibration bias or DC offset compensation;

\#BPSK modulation format for BPSK MMICs and QPSK modulation for IQ MMICs with a 1-Msps symbol rate.

high-data-rate operation of the arbitrary waveform generators and the nonlinearity of the single-to-differential circuit.

The vector signal characterizations were also performed at 50, 60, 77, and $110 \mathrm{GHz}$ for the BPSK and the IQ modulators. The performance summary of the MMICs is summarized in Table III. As can be observed, the modulator MMICs demonstrated an EVM degradation of less than $12 \%$, an LO rejection of more than $15 \mathrm{~dB}$ and an ACPR of better than $-21 \mathrm{dBc}$ between $50-110 \mathrm{GHz}$.

\section{CONCLUSION}

Reflection-type broad-band HBT BPSK and IQ modulator MMICs have been reported in this paper. These MMICs are suitable for broad-band digital modulations due to their wide bandwidth, low EVM degradation, good LO rejection, and ACPR performance. A cold-mode HBT device model is also developed and verified by comparing simulation and measurement results. The analysis and design equations of imbalance effects for a reflection-type modulator are developed to obtain the circuit design rules. For circuit characterization, the equations of carrier suppression and sideband suppressions for reflection-type IQ modulators are investigated and the sideband suppression measurements are also performed. Simultaneously, these MMICs are evaluated successfully by the vector signal measurement, and the EVM measurement results are also evaluated with the sideband suppression characterization. Therefore, these MMICs provide a low-cost solution and broad-band modulator for MMW applications in the frequency band of 50-110 GHz.

\section{ACKNOWLEDGMENT}

The authors gratefully acknowledge the WIN Semiconductors Corporation, Taoyuan, Taiwan, R.O.C., for the fabrication of the MMIC chips. The authors would also like to thank G. G. Boll, GGB Inc., Naples, FL, for providing the $W$-band probes for chip on-wafer testing.

\section{REFERENCES}

[1] R. Desrosiers, J. Cowles, C. Hornbuckle, A. Gutierrez-Aitken, and J. Becker, "Monolithic $14 \mathrm{GHz}$ widerband InP HBT BPSK modulator," in Proc. Gallium Arsenide Integrated Circuit Symp., 1998, pp. 135-138.

[2] I. Telliez, A.-M. Couturier, C. Rumelhard, C. Versnaeyen, P. Champion, and D. Fayol, "A compact monolithic microwave demodulator-modulator for 64-QAM digital radio links," IEEE Trans. Microwave Theory Tech., vol. 39, pp. 1947-1954, Dec. 1991.

[3] G. Samuel Dow, J. Yang, and K.-H. Yen, "Vector signal measurement for $38 \mathrm{GHz}$ digital radio applications," Microwave J., vol. 42, no. 10, pp. 94-106, Oct. 1999.
[4] S. G. Dow, J. M. Yang, K. H. Yen, R. Matreci, E. Spotted-Elk, S. Pettis, and L. Trinh, "Vector signal characterization of $38 \mathrm{GHz}$ power amplifier with 100 Mbps QPSK modulation," in IEEE MTT-S Int. Microwave Symp. Dig., 2000, pp. 1847-1850.

[5] A. Alexanian, M. Wu, A. Burgess, Y. Wei, and X. Xhang, "A SiGe transceiver chipset for $100 \mathrm{Mbps} / 1 \mathrm{Gbps}$ digital communication over cable system," in Proc. IEEE Radio Frequency Integrated Circuits Symp., 2002, pp. 119-122.

[6] A. Weetzei, "A stable 250 to $4000 \mathrm{MHz}$ GaAs IQ modulator IC," in IEEE Int. Solid-State Circuits Conf. Dig., 1997, pp. 364-365.

[7] A. Boveda, F. Orilgoso, and J. I. Alonso, "A $0.7-3 \mathrm{GHz}$ GaAs QPSK/QAM direct modulator," IEEE J. Solid-State Circuits, vol. 28, pp. 1340-1349, Dec. 1993.

[8] G. Brenna, D. Tschopp, D. Pfaff, and Q. Huang, "A 2 GHz direct conversion WCDMA modulator in $0.25 \mu \mathrm{m}$ CMOS," in IEEE Int. Solid-State Circuits Conf. Dig., vol. 2, 2002, pp. 232-235.

[9] A. Loke and F. Ali, "Direct conversion radio for digital mobile phonesdesign issues, status, and trends," IEEE Trans. Microwave Theory Tech., vol. 50, pp. 2422-2435, Nov. 2002.

[10] D. C. W. Lo, H. Wang, B. R. Allen, G. S. Dow, K. W. Chang, M. Biedenbender, R. Lai, S. Chen, and D. Yang, "Novel monolithic multifunctional balanced switching low-noise amplifiers," IEEE Trans. Microwave Theory Tech., vol. 42, pp. 2629-2634, Dec. 1994.

[11] T. Lodhi, D. L. Edgar, H. McLelland, S. Ferguson, K. Elgaid, C. R. Stanley, and I. G. Thayne, "A $77 \mathrm{GHz}$ coplanar waveguide MMIC BPSK vector modulator realized using InP technology," in IEEE Gallium Arsenide Integrated Circuit Symp. Dig., 2000, pp. 183-186.

[12] A. Ashtiani, S.-I. Nam, A. d'Espona, S. Lucyszyn, and I. D. Robertson, "Direct multilevel carrier modulation using millimeter-wave balanced vector modulators," IEEE Trans. Microwave Theory Tech., vol. 46, pp. 2611-2619, Dec. 1998.

[13] S. Nam, N. Shala, K. S. Ang, A. E. Ashtiani, T. Gokdemir, I. D. Robertson, and S. P. Marsh, "Monolithic millimeter-wave balanced bi-phase amplitude modulator in GaAs/InGaP HBT technology," in IEEE MTT-S Int. Microwave Symp. Dig., vol. 1, 1999, pp. 243-246.

[14] A. E. Ashtiani, T. Gokdemir, A. Vilches, Z. Hu, I. D. Robertson, and S. P. Marsh, "Monolithic GaAs/InGaP HBT balanced vector modulator for millimeter-wave wireless systems," in IEEE Radio Frequency Integrated Circuits Symp. Dig., 2000, pp. 187-190.

[15] H.-Y. Chang, H. Wang, Y.-C. Wang, P.-C. Chao, C.-H. Chen, and D.-C. Niu, "A 45-90 GHz BPSK modulator using HBT technology," in Proc. Asia-Pacific Microwave Conf., vol. 1, Kyoto, Japan, Nov. 2002, pp. 60-62.

[16] H.-Y. Chang, T.-W. Huang, H. Wang, Y.-C. Wang, P.-C. Chao, and C.-H. Chen, "A broadband HBT MMIC IQ modulator and millimeter-wave vector signal characterization," in IEEE MTT-S Int. Microwave Symp. Dig., June 2003, pp. 99-102.

[17] C.-J. Wei and J. C. M. Huwang, "Direct extraction of equivalent circuit parameters for heterojunction bipolar transisitors," IEEE Trans. Microwave Theory Tech., vol. 43, pp. 2035-2039, Sept. 1995.

[18] D. A. Teeter and W. R. Curtice, "Comparison of hybrid pi and tee HBT circuit topologies and their relationship to large signal modeling," in IEEE MTT-S Int. Microwave Symp. Dig., vol. 2, 1997, pp. 375-378.

[19] S. Bousnina, P. Mandeville, A. B. Kouki, R. Surridge, and F. M. Ghannouchi, "Direct parameter-extraction method for HBT small-signal model," IEEE Trans. Microwave Theory Tech., vol. 50, pp. 529-536, Feb. 2002.

[20] Y. Suh, E. Seok, J.-H. Shin, B. Kim, D. Heo, A. Raghavan, and J. Laskar, "Direct extraction method for internal equivalent circuit parameters of HBT small-signal hybrid- $\pi$ model," in IEEE MTT-S Int. Microwave Symp. Dig., vol. 3, 2000, pp. 1401-1403.

[21] GaAs $1 \mu \mathrm{m}$ HBT Model Handbook 1.0, WIN Semiconduct. Corporation, Taoyuan, Taiwan, R.O.C., July 2001. 
[22] Sonnet User's Manual, Release 6.0, Sonnet Software Inc., Liverpool, NY, Apr. 1999.

[23] L. Sundstrom, M. Faulkner, and M. Johansson, "Quantization analysis and design of a digital predistortion linearizer for RF power amplifiers," IEEE Trans. Veh. Technol., vol. 45, pp. 707-719, Nov. 1996.

[24] S. A. Maas, B. L. Nelson, and D. L. Tait, "Intermodulation in heterjunction bipilar transistors," IEEE Trans. Microwave Theory Tech, vol. 40, pp. 442-448, Mar. 1992.

[25] B. Li and S. Prasad, "Intermodulation analysis of the collector-up InGaAs/InAlAs/InP HBT using volterra series," IEEE Trans. Microwave Theory Tech., vol. 46, pp. 1321-1323, Sept. 1998.

[26] C. Lanschutzer, A. Springer, L. Maurer, Z. Boos, and R. Weigel, "A digital adaptive feedback loop for local oscillator leakage cancellation and DC offset compensation," in Proc. Asia-Pacific Microwave Conf., vol. 1, Kyoto, Japan, Nov. 2002, pp. 409-412.

[27] L. Der and B. Razavi, "A 2-GHz CMOS image-reject receiver with LMS calibration," IEEE J. Solid-State Circuits, vol. 38, pp. 167-175, Feb. 2003.

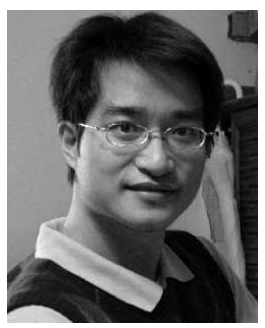

Hong-Yeh Chang ( $\left.\mathrm{S}^{\prime} \mathrm{O2}\right)$ was born in Kinmen, $\mathrm{Fu}$ jian, China, in 1973. He received the B.S. and M.S degrees in electric engineering from the National Center University, Chung-Li, Taiwan, R.O.C., in 1995 and 1997, respectively, and is currently working toward the Ph.D. degree in communication engineering at the National Taiwan University, Taipei, Taiwan, R.O.C.

His research interests include microwave and MMW circuit designs.

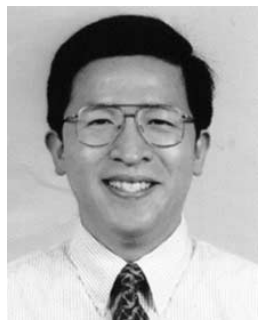

Tian-Wei Huang (S'91-M'98-SM'02) received the B.S. degree in electrical engineering from the National Cheng Kung University, Tainan, Taiwan, R.O.C., in 1987, and the M.S. and Ph.D. degree in electrical engineering from the University of California at Los Angeles (UCLA), in 1990 and 1993, respectively.

In 1993, he joined the TRW RF Product Center, Redondo Beach, CA. His research has focused on the design and testing of MMICs and RF integrated circuits (RFICs). From 1998 to 1999 , he was with $\mathrm{Lu}-$ cent Technologies, where he was involved with local multipoint distribution system (LMDS) fixed wireless systems. From 1999 to 2002, he was with Cisco Systems, where he was involved with RF/wireless system testing. In August 2002, he joined the faculty of the Department of Electrical Engineering, National Taiwan University. His current research interests are MMIC/RFIC design, packaging, and RF system-on-chip (SOC) integration.

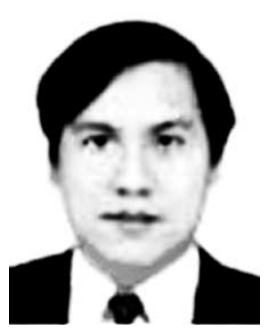

Huei Wang (S'83-M'87-SM'95) was born in Tainan, Taiwan, R.O.C., on March 9, 1958. He received the B.S. degree in electrical engineering from the National Taiwan University, Taipei, Taiwan, R.O.C., in 1980, and the M.S. and Ph.D. degrees in electrical engineering from Michigan State University, East Lansing, in 1984 and 1987, respectively.

During his graduate study, he was engaged in research on theoretical and numerical analysis of EM radiation and scattering problems. He was also involved in the development of microwave remote detecting/sensing systems. In 1987, he joined the Electronic Systems and Technology Division, TRW Inc. He was a Member of the Technical Staff and Staff Engineer responsible for MMIC modeling of computer-aided design (CAD) tools, MMIC testing evaluation, and design. He then became the Senior Section Manager of the Millimeter Wave Sensor Product Section, RF Product Center, TRW Inc. In 1993, he visited the Institute of Electronics, National Chiao-Tung University, Hsin-Chu, Taiwan, R.O.C., and taught MMIC-related topics. In 1994, he returned to TRW Inc. In February 1998, he joined the faculty of the Department of Electrical Engineering, National Taiwan University, as a Professor.

Dr. Wang is a member of Phi Kappa Phi and Tau Beta Pi.
Yu-Chi Wang received the B.S. degree in physics from the National Central University, Chung-Li, Taiwan, R.O.C., in 1989, and the M.S. and Ph.D. degrees in materials science and engineering from Rutgers University, New Brunswick, NJ, in 1994 and 1998, respectively. His doctoral dissertation concerned the device and circuit design, metal-beam-epitaxy growth, fabrication, and characterization of $\mathrm{In}_{0.5}\left(\mathrm{Al}_{\mathrm{x}} \mathrm{Ga}_{1-\mathrm{x}}\right)_{0.5} \mathrm{P}$ power HEMTs.

In 1998, he joined Bell Laboratories, Lucent Technologies, Murray Hill, NJ, where he was a Member of Technical Staff, involved in the device design and process development of GaAs metal-oxide-semiconductor field-effect transistors, 0.1- $\mu \mathrm{m}$ InAlAs-InGaAs HEMTs, and high-performance InP-InGaAs-InP double HBTs for 40-Gb/s lightwave circuits. In December 1999, he joined the WIN Semiconductors Corporation, Taoyuan, Taiwan, R.O.C., where he was responsible for the InGaP HBT technology development for cellular handset power amplifiers and wireless local area network (WLAN) power amplifiers. He is currently the Director of the Technology Development Division, WIN Semiconductors Corporation.

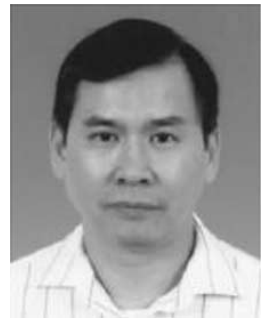

Pane-Chane Chao (S'80-M'82-SM'88) received the $\mathrm{Ph} . \mathrm{D}$. degree in electrical engineering from Cornell University, Ithaca, NY, in 1982.

$\mathrm{He}$ then joined the General Electric (GE) Aerospace Division, Syracuse, NY. While with GE, he was responsible for GaAs MESFET and HEMT MMIC process technology development. He was the first to implement the low-resistance T-gate technology on GaAs HEMTs - setting an industry standard. Working with the University of Illinois in 1985, he and his team successfully demonstrated the world's first $60-\mathrm{GHz}$ power pseudomorphic high electron-mobility transistor (pHEMT), opening the door for both military and commercial applications of this technology. In 1996, he joined Lockheed-Martin, Nashua NH, where he managed the Advanced HEMT MMIC Process Development Group to explore GaAs pHEMT, InP HEMT, metamorphic HEMT, and GaN HEMT technologies. In September 2000, he joined the WIN Semiconductors Corporation, Taoyuan, Taiwan, R.O.C., where, as a Chief Technology Officer (CTO) and Senior Vice President, he leads 6-in GaAs HBT and pHEMT technology development and manufacturing. In 2001, his team successfully demonstrated the first 6-in $0.15-\mu \mathrm{m}$ pHEMT wafer in the world. He has authored and coauthored over 150 technical papers.

Dr. Chao is a BAE Engineering Fellow. He currently serves as a Technical Program Committee member of the International GaAs ManTech Conference.

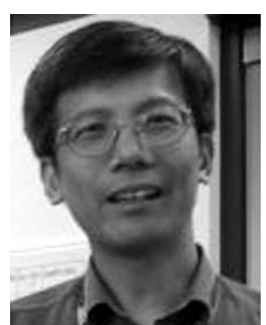

R.O.C.
Chung-Hsu Chen received the Ph.D. degree from the University of Minnesota at Minneapolis-St. Paul, in 1986.

He was with Honeywell for three years, where he was invovled with GaAs processing and modeling. He then spent approximately 11 years with the TRW GaAs Foundry Laboratory, where he was involved in product engineering, power devices characterization, and power-match tuning, where he was responsible for life test and layout. He is currently with the WIN Semiconductors Corporation, Taoyuan, Taiwan, 